\title{
Health Risk Assessment from Exposure to Heavy Metals in Surface and Groundwater Resources within Barkin Ladi, North Central Nigeria
}

\author{
Joyce Ayuba Ramadan'1, Ahmad Isah Haruna² \\ ${ }^{1}$ Department of Minerals \& Petroleum Resources Engineering, Plateau State Polytechnic, Barkin Ladi, Nigeria \\ ${ }^{2}$ Department of Applied Geology, Abubakar Tafawa Balewa University, Bauchi, Nigeria \\ Email:ramadanjoyce@gmail.com, iaharuna@atbu.edu.ng
}

How to cite this paper: Ramadan, J. A., \& Haruna, A. I. (2019). Health Risk Assessment from Exposure to Heavy Metals in Surface and Groundwater Resources within Barkin Ladi, North Central Nigeria. Journal of Geoscience and Environment Protection, 7, 1-21.

https://doi.org/10.4236/gep.2019.72001

Received: December 31, 2018

Accepted: January 30, 2019

Published: February 2, 2019

Copyright $\odot 2019$ by author(s) and Scientific Research Publishing Inc. This work is licensed under the Creative Commons Attribution International License (CC BY 4.0).

http://creativecommons.org/licenses/by/4.0/

\begin{abstract}
Concentration of heavy metals from surface and groundwater within the communities were determined in order to assess the health risks linked to the use/consumption of same. Furthermore, the cancer risk for adults in surface water revealed medium to high cancer risk for arsenic (As); low to medium cancer risk for cadmium (Cd) and very high cancer risk for chromium (Cr). In addition, the cancer risk for adults in groundwater is as follows: high cancer risk for As; low cancer risk for $\mathrm{Cd}$ and very high cancer risk for $\mathrm{Cr}$ while that for children is very high cancer risk for As and $\mathrm{Cr}$ and then low cancer risk for $\mathrm{Cd}$. The findings from this research confirm that the inhabitants within the study area are at direct health risk (carcinogenic and non-carcinogenic) due to the alternate use of surface and groundwater without any form of treatment.
\end{abstract}

\section{Keywords}

Health-Risk, Heavy Metals, Assessment, Carcinogenic, Non-Carcinogenic

\section{Introduction}

The health risks assessment associated with the consumption of water as well as use for domestic and irrigation purposes from abandoned mine ponds, streams and groundwater resources, was conducted. This was considered within Barkin ladi and surrounding localities which forms part of the Jos Plateau where mechanized tin mining activities took place for over seven 
decades (Ramadan \& Haruna, 2018) and small scale mining activities are still on-going.

Generally, tin mining activities have the tendency of contributing a considerable quantity of heavy metals to the environment. Although, some of these metals could be essential to humans, they also could be toxic to same when they exceed the maximum admissible values (Adamu et al., 2014). And the inhabitants residing around these mine sites are particularly at risk of such exposure. Human exposure to such toxic heavy metals could be direct or indirect (Ayantobo et al., 2014). Direct pathways are through inhalation, dermal absorption or water ingestion while indirect exposure occurs as a result of bioaccumulation (USEPA, 2001). The links between environment and health are particularly important for subsistence populations who are heavily dependent on the local environment for their food supply (Fordyce et al., 1999) and most of the settlements within the study area are as such. Heavy metals such as arsenic, mercury and cadmium have drawn great attention in the globe and are widely distributed in the environment, including sources of drinking water through which they could cause damage to the liver, kidney, digestive and nervous system (Montuori et al., 2013).

The key issues of concern within the study area are the fact that inhabitants depend solely on hand dug wells, boreholes, streams and abandoned mine ponds for water utilized for domestic and irrigation purposes. Therefore, the principal objective of this research is to investigate the occurrence and concentration of heavy metals in surface and groundwater and further evaluate the health risk to residents within the study area.

\section{Physical Setting}

The area of investigation is located in north-central Nigeria and is geographically bounded between latitudes $9^{\circ} 32^{\prime} 28^{\prime \prime} \mathrm{N}$ and $9^{\circ} 43^{\prime} 29^{\prime \prime} \mathrm{N}$ and longitudes $8^{\circ} 52^{\prime} 20^{\prime \prime} \mathrm{E}$ and $8^{\circ} 56^{\prime} 12^{\prime \prime}$ (Figure 1). It encompasses communities like Bisichi, Kuru Jenta, Foron, Heipang, Kassa and Barkin Ladi (Figure 1) and covers a total land area of about $198 \mathrm{~km}^{2}$. It is generally accessible through the Mararaban Jama'a-Mangu express road with some tarred feeder roads and footpaths linking the various communities.

The study area is characterized by the tropical climatic condition prevailing on the Jos Plateau. Here the climate is controlled by two seasons, namely, the wet and dry seasons. The former prevails between the months of April and October, while the latter starts in the month of November and ends in the month of March. The mean annual rainfall for Barkin ladi and its environs is about 1855 $\mathrm{mm}$. The vegetation of the study area is that of the guinea savannah characterized by tall grasses interspersed with trees (Iloeje, 1981). The common trees are acacias, baobab and shea butter (Ajayi, 2003).

The rocks found within the study area consists of the Newer Basalts, Younger Granites and Older Granitic rocks. 


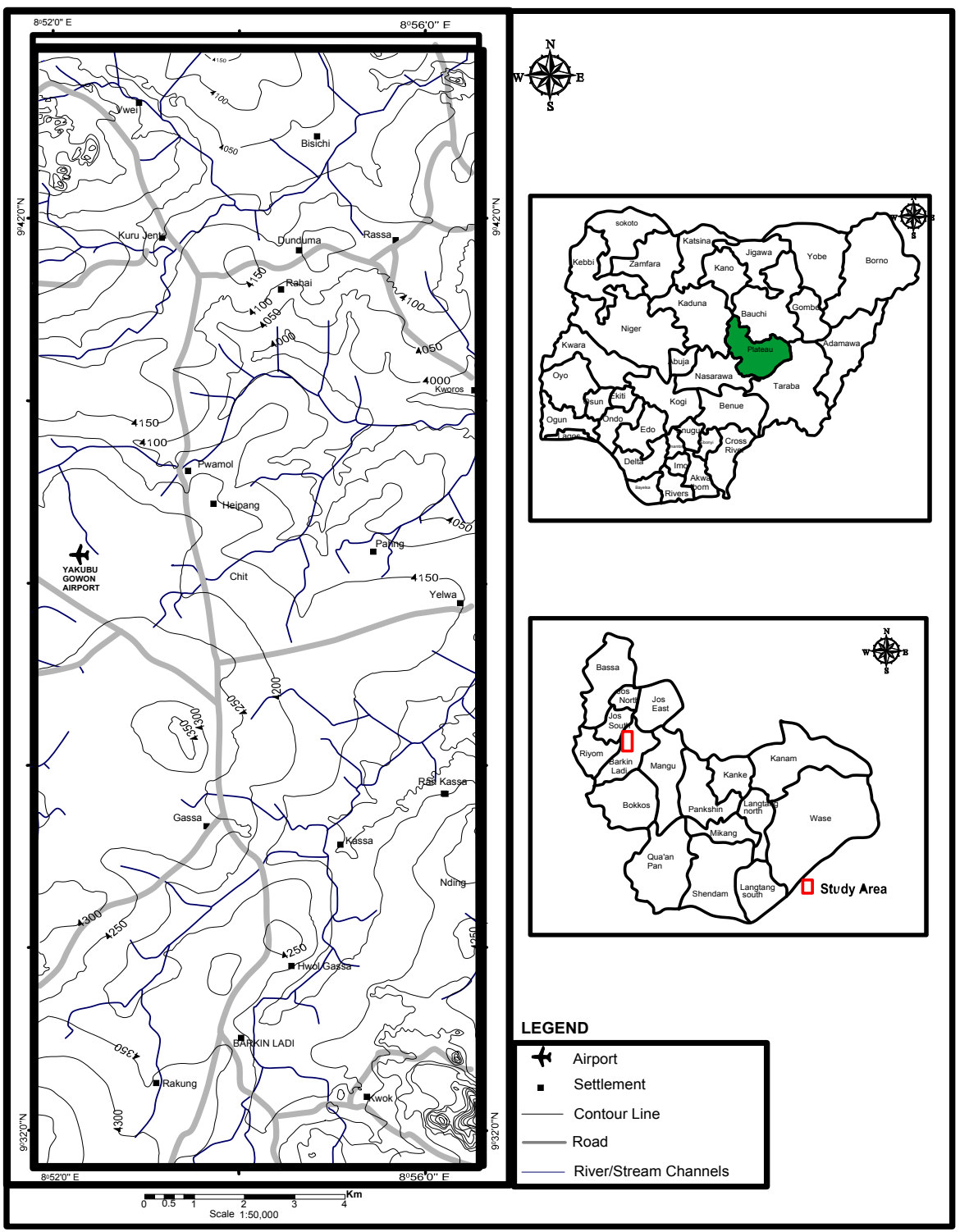

Figure 1. Location map of the study area (modified from MacLeod \& Berridge, 1971).

\section{Methods and Materials}

\subsection{Questionnaire Administration}

A total of two hundred and seventeen (217) questionnaires were administered through personal one on one interview with residents who have lived most part of their lives in the direct communities within the study area. Personal background and general information like their age, the length of time they have lived in those communities, their source of water supply, the prevalent illnesses experienced over time of the people were gathered.

\subsection{Water Sampling}

A total number of forty six (46) water samples were assembled within the study area (Figure 2) at the peak of the dry season in order to avoid dilution by the 


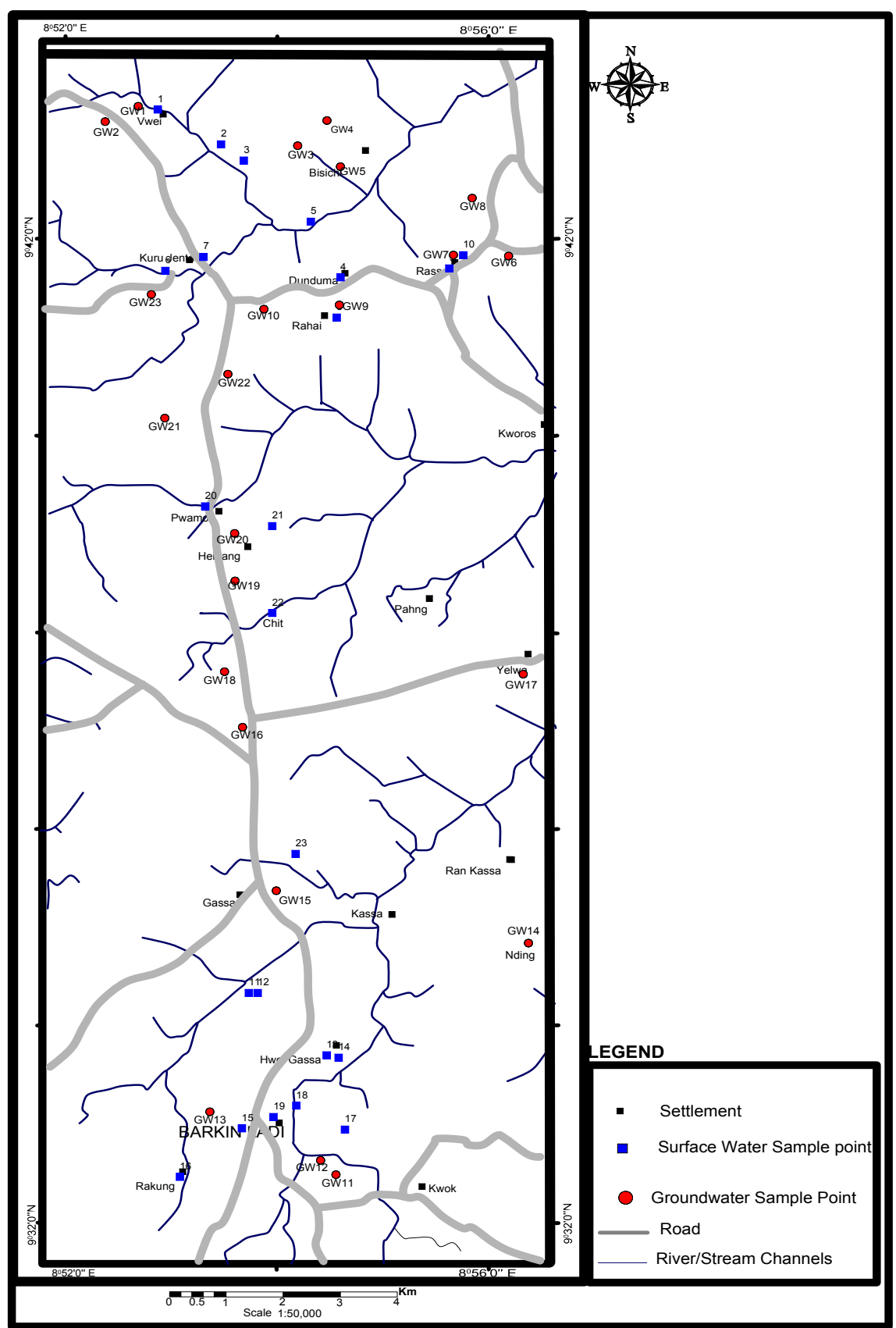

Figure 2. Map of the study area showing water sample locations (modified from MacLeod \& Berridge, 1971).

rain waters. A good number of the mine ponds are situated along stream channels, twenty three (23) samples of water from these were collected, as well as from flowing stream channels, while another set of twenty three (23) samples were drawn from hand dug wells and boreholes which are mostly sited within residential settlements. These were collected in polyethylene containers previously washed and repeatedly rinsed with distilled water and kept in sealed. At the point of sampling, the containers were rinsed twice with the sample to be collected before the final collection. On-site measurements of $\mathrm{pH}$, temperature, 
conductivity and Total Dissolved Solids (TDS) were obtained using a potable handheld waterproof pH/EC/Temp/TDS tester. Furthermore, the Garmin Global Positioning System (GPS) was used to locate positions where each sample was collected.

The filtered and acidified water samples were analyzed at Bureau Veritas Minerals (BVM) laboratory, Vancouver, Canada; using the Inductively Coupled Plasma Mass Spectrometry (ICP-MS) method of analysis.

\subsection{Human Health Risk Assessment}

Human exposure to heavy metals is through several pathways including oral, inhalation and dermal (Elumalai et al., 2017). Risk assessment is a function of the hazard and exposure (Adamu et al., 2015) and is defined as the process of estimating the probability of occurrence of an event and the probable magnitude of adverse health effects on human exposure to environmental hazards over a specified period of time. The health risk assessment of each potentially toxic metal is usually based on the quantification of the risk level and is expressed in terms of a carcinogenic or a non-carcinogenic health risk (Lim et al., 2008). The two principal toxicity risk factors evaluated are the slope factor (SF) for carcinogen risk characterization and the reference dose (RfD) for non-carcinogen risk characterization.

The toxicity indices of each potentially toxic metal are shown in Table 1 (USEPA IRIS, 2011). The estimations of the magnitude, frequency and duration of human exposure to each potentially toxic metal in the environment are reported as average daily dose (ADD) (Siriwong, 2006) and calculated using:

$$
\mathrm{ADD}=\frac{\mathrm{C} \times \mathrm{IR} \times \mathrm{ED} \times \mathrm{EF}}{\mathrm{BW} \times \mathrm{AT} \times 365}
$$

where $\mathrm{ADD}$ is the average daily dose $(\mathrm{mg} / \mathrm{kg} / \mathrm{day}), \mathrm{C}$ is the concentration $(\mathrm{mg} / \mathrm{L})$

Table 1. Toxicity Responses to Heavy Metals as the Oral Reference Dose (Rfd) and Oral Slope Factor (SF) (USEPA IRIS, 2011).

\begin{tabular}{cccc}
\hline $\mathrm{S} / \mathrm{n}$ & Metals & $\begin{array}{c}\text { Oral RfD } \\
(\mathrm{mg} / \mathrm{kg} / \mathrm{day})\end{array}$ & $\begin{array}{c}\text { Oral SF } \\
(\mathrm{mg} / \mathrm{kg} / \mathrm{day})\end{array}$ \\
\hline 1 & As & $3.0 \times 10^{-4}$ & 1.5 \\
2 & $\mathrm{Cd}$ & $5.0 \times 10^{-4}$ & 0.380 \\
3 & $\mathrm{Cr}$ & $3.0 \times 10^{-3}$ & 41.0 \\
4 & $\mathrm{Cu}$ & $4.0 \times 10^{-2}$ & n.d \\
5 & $\mathrm{~Pb}$ & $3.5 \times 10^{-3}$ & n.d \\
6 & $\mathrm{Ni}$ & $2.0 \times 10^{-2}$ & n.d \\
7 & $\mathrm{Zn}$ & 0.3 & n.d \\
8 & $\mathrm{Fe}$ & $7.0 \times 10^{-1}$ & n.d \\
9 & $\mathrm{Mn}$ & $1.4 \times 10^{-1}$ & n.d \\
10 & $\mathrm{Co}$ & $3.0 \times 10^{-4}$ & n.d \\
\hline
\end{tabular}


of heavy metal measured, IR is the water intake rate $(3.45$ and $2.0 \mathrm{~L} /$ day for adults and children respectively) (Apambire et al., 1997; Roychowdhury et al., 2003), $\mathrm{EF}$ is the exposure frequency (365 days/year), ED is the exposure duration (70 years in the case of adults and 10 years for children), BW is the average body weight [60 kg (adults) and $25 \mathrm{~kg}$ (children)], and AT is the average time (25,550 days, i.e. 70 years $\times 365$ days/year (adults) and 3650 days, i.e. 10 years $\times 365$ days/year (children) (Wongsasuluk et al., 2014; Roychowdhury et al., 2003).

The input parameters in ADD formula are shown in Table 2.

The final stage of health risk assessment is the risk characterization. In this stage, the exposure and dose response assessments are integrated to yield probabilities of effects occurring in human beings under specific exposure conditions (Ayantobo et al., 2014). The extent of harm sustained is expressed in terms of hazard quotient as follows:

$$
\text { Hazard quotient }(\mathrm{HQ})=\frac{\mathrm{ADD}}{\mathrm{RfD}}
$$

where $\mathrm{ADD}$ and $\mathrm{RfD}$ are in $\mathrm{mg} / \mathrm{kg}$-day. In the case of hazard index (HI) which is the sum of individual HQ's representing a mixture of chemicals:

$$
\text { Hazard Index }(\mathrm{HI})=\sum \mathrm{HQ}_{i}
$$

The carcinogenic risks are expressed in terms of the probability that one may develop cancer at a given lifetime exposure level. The cancer risk probability is determined from the slope factor (SF) which denotes the probability of developing cancer per unit exposure level if $\mathrm{mg} / \mathrm{kg} /$ day and its data can be obtained from IRIS database as displayed in Table 3. Cancer risk is then calculated as follows:

$$
\text { Cancer risk }=\mathrm{ADD} \times \mathrm{SF}
$$

The scale for classification is presented in Table 3 , alongside the non-carcinogenic risk evaluation.

\section{Results and Discussions}

\subsection{Questionnaire Analysis}

Inhabitants living within the study area were interviewed through the use of investigation technique in a random manner. A total of two hundred and seventeen

Table 2. Parameters to Characterize the ADD Value.

\begin{tabular}{ccccc}
\hline S/n & Exposure parameters & Symbols & Unit & Value \\
\hline 1 & Conc. Of Element In Water & C & $\mathrm{mg} / \mathrm{l}$ & As measured (from this study) \\
2 & Exposure Duration & ED & Years & 70 for adults and 10 for children \\
3 & Exposure Frequency & EF & Days/year & 365 \\
4 & Average Time & AT & days & 25,550 for adults and 3650 for children \\
5 & Body Weight & BW & Kg & 60 for adults and 25 for children \\
6 & Ingestion Rate & IR & $1 /$ day & 2 for children and 3.45 for adults
\end{tabular}


Table 3. Scales for non-carcinogenic and carcinogenic risk assessment (USEPA, 1999).

\begin{tabular}{ccccc}
\hline Risk level & HQ/HI & $\begin{array}{c}\text { Adverse } \\
\text { Non-carcinogenic Risk }\end{array}$ & $\begin{array}{c}\text { Calculated cases of Cancer } \\
\text { Occurrence }\end{array}$ & Cancer Risk \\
\hline 1 & $<0.1$ & Negligible & $<1$ per $1,000,000$ inhabitants & Very low \\
2 & $\geq 0.1<1$ & Low & $\begin{array}{c}>1 \text { per } 1,000,000 \text { inhabitants } \\
<1 \text { per } 100,000 \text { inhabitants }\end{array}$ & Low \\
& & & $>1$ per 100,000 inhabitants & Medium \\
3 & $\geq 1<4$ & Medium & $<$ per 10,000 inhabitants & \\
& & & $>1$ per 10,000 inhabitants & High \\
4 & $\geq 4$ & High & $<1$ per 1000 inhabitants & Very high \\
& & & $>1$ per 1000 inhabitants &
\end{tabular}

(217) questionnaires were administered. Of special relevance to this research are the source(s) of water for consumption and domestic use as well as the prevalent illnesses experienced by the same population.

From the analyses of all the questionnaires, $22.6 \%$ of the interviewers obtain water from Hand Dug Wells (HDW), 5.6\% from streams and 2.4\% from Boreholes $(\mathrm{BH})$. However, most of the inhabitants collect water for consumption and domestic purposes from a combination of two or three sources earlier mentioned because most of these sources are seasonal in their supply. 19.3\% source water from a combination of streams, HDW, BH and mine ponds (MP). Similarly, $12.7 \%$ obtain water from HDW and MP; $10.8 \%$ acquire water from stream channels, HDW and MP; and 10.3\% combine sources from streams, BH and HDW. In the same way, $7.5 \%$ of the inhabitants interviewed collect water from streams and HDW; $4.7 \%$ from HDW and $\mathrm{BH}$ and $4.2 \%$ collect water from HDW, BH and MP (Figure 3). Consequently, rendering them vulnerable of being exposed to heavy metals and at risk of manifesting adverse health effects over a period of time.

According to the data analyses, the prevalent illnesses experienced by the inhabitants within and around the study area, $1.9 \%$ have come down with cancers of various forms, $0.5 \%$ with skin disease; while a greater part of the population (27.7\%) suffer diseases like typhoid and/or diarrhea and/or dysentery and/or cholera captured as "others" (Figure 4). Other records include cases of cancers and kidney disease as $0.5 \% ; 4.7 \%$ of the population suffer cancers and others; 24.4\% have experienced cancers, skin diseases and others; $4.2 \%$, skin diseases, tuberculosis and others; while $4.7 \%$ of those interviewed have had relations or themselves experienced cancers, skin disease, kidney disease and others; $1.4 \%$ with skin disease and tuberculosis; $0.9 \%$, kidney disease and others and $0.9 \%$ have been ill with cancers, skin diseases, kidney disease, tuberculosis and others. The manifestations of some of these diseases could be linked to the exposure of the potentially heavy metals.

\subsection{Concentrations of Heavy Metals in Surface and Groundwater}

The mean concentrations of heavy metals determined in surface water within 


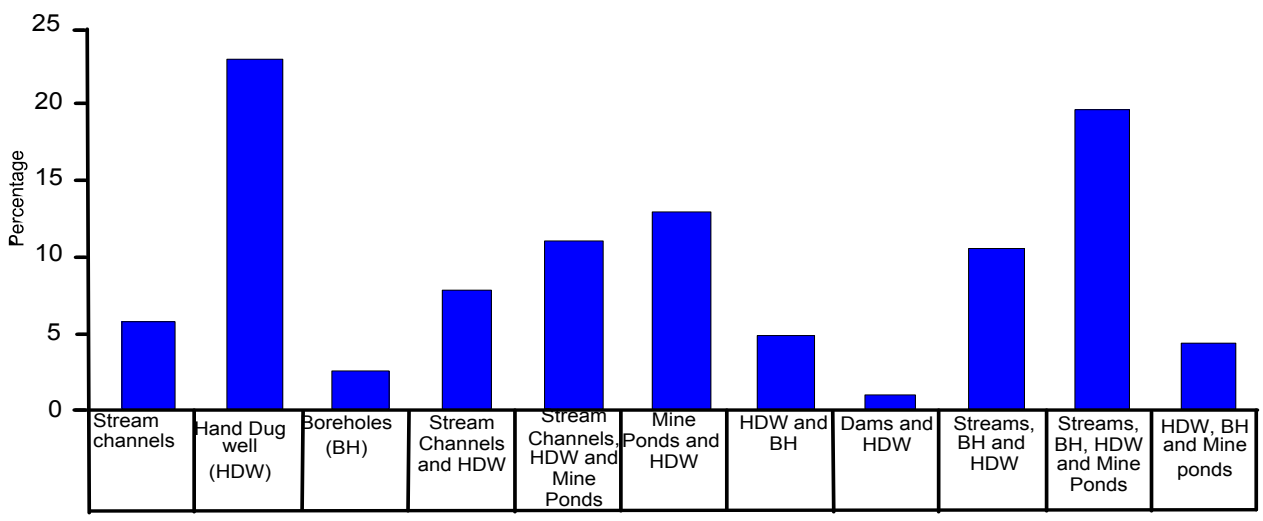

Figure 3. Bar chart showing the percentage of the source of water for use within the study area.

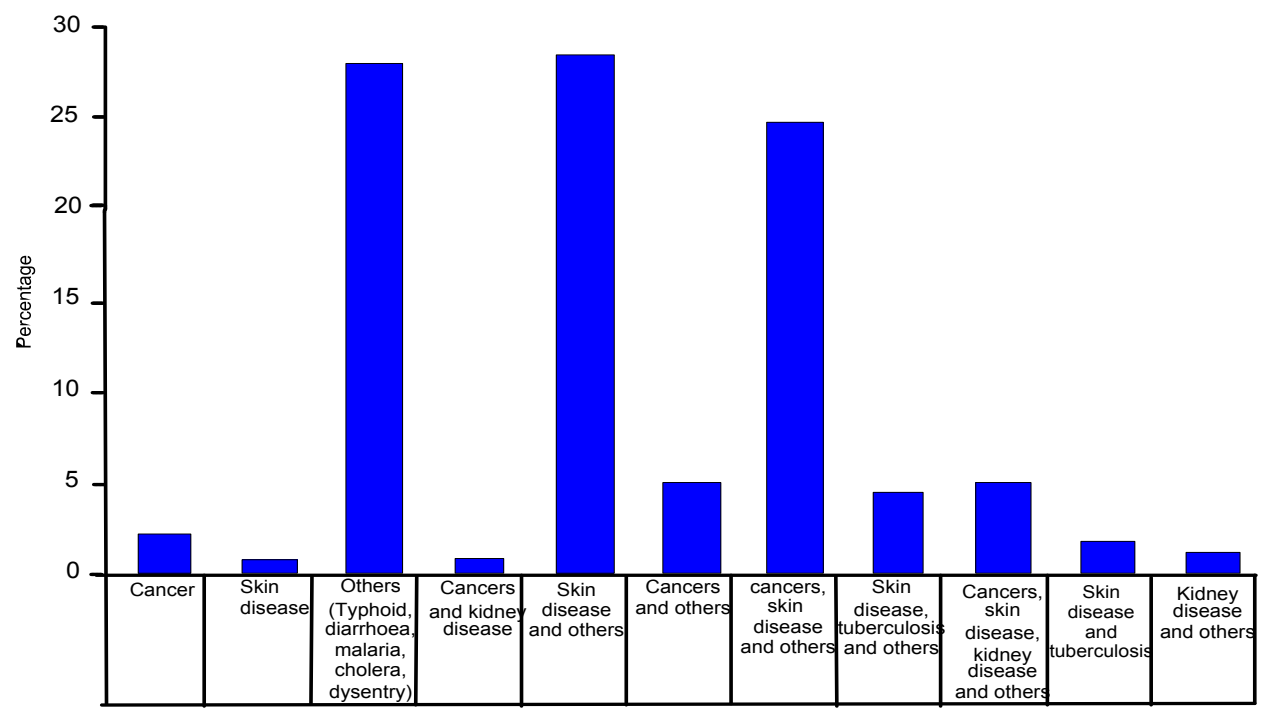

Figure 4. Bar chart showing the Prevalent Diseases experienced by Inhabitants within the Study Area.

the study area were in the following order Fe $(2.504 \mathrm{mg} / \mathrm{l})>\mathrm{Mn}(0.2990 \mathrm{mg} / \mathrm{l})>$ $\mathrm{Zn}(0.0604 \mathrm{mg} / \mathrm{l})>\mathrm{Cu}(0.0139 \mathrm{mg} / \mathrm{l})>\mathrm{Pb}(0.0137 \mathrm{mg} / \mathrm{l})>\mathrm{Co}(0.00506 \mathrm{mg} / \mathrm{l})>$ $\mathrm{Ni}(0.0039 \mathrm{mg} / \mathrm{l})=\mathrm{Cr}(0.0039 \mathrm{mg} / \mathrm{l})>$ As $(0.0002 \mathrm{mg} / \mathrm{l})>\mathrm{Cd}(0.00007 \mathrm{mg} / \mathrm{l})$. Health hazards were identified in some surface water locations for instance iron (Fe) exceeds the Nigerian standard guideline value in all locations except SW8, SW13, SW15 and SW19. Also, manganese (Mn) exceeds the standard guideline value in SW6, SW9, SW10, SW21 and SW 23 as well as lead (Pb) in SW1, SW2, SW7, SW9, SW10 and SW16 as shown in Table 4.

In groundwater, the mean concentration of heavy metals is the following order: $\mathrm{Fe}(0.339 \mathrm{mg} / \mathrm{l})>\mathrm{Zn}(0.0518 \mathrm{mg} / \mathrm{l})>\mathrm{Mn}(0.03512 \mathrm{mg} / \mathrm{l})>\mathrm{Cu}(0.0133$ $\mathrm{mg} / \mathrm{l})>\mathrm{Ni}(0.0026 \mathrm{mg} / \mathrm{l})>\mathrm{Pb}(0.0020 \mathrm{mg} / \mathrm{l})>\mathrm{Co}(0.00102 \mathrm{mg} / \mathrm{l})>\mathrm{As}(0)=\mathrm{Cd}$ (0). It was also observed that the Iron $(\mathrm{Fe})$ concentration surpasses the Nigerian standard guideline in GW9, GW11, GW13, GW14 and GW 17. Likewise, Lead $(\mathrm{Pb})$ in GW22 as displayed in Table 5, which pose a vulnerability risk to the inhabitants. 
Table 4. Heavy metal concentration and physical parameters in surface water.

\begin{tabular}{|c|c|c|c|c|c|c|c|c|c|c|c|c|c|c|c|c|c|c|}
\hline $\begin{array}{l}\text { Sample } \\
\text { location }\end{array}$ & $\begin{array}{l}\text { Sample } \\
\text { code No }\end{array}$ & Des. & Long & Lat & $\begin{array}{c}\mathrm{As} \\
\mathrm{mg} / \mathrm{l}\end{array}$ & $\begin{array}{l}\mathrm{Co} \\
\mathrm{mg} / \mathrm{l}\end{array}$ & $\begin{array}{c}\mathrm{Cd} \\
\mathrm{mg} / \mathrm{l}\end{array}$ & $\begin{array}{c}\mathrm{Cr} \\
\mathrm{mg} / \mathrm{l}\end{array}$ & $\begin{array}{c}\mathrm{Cu} \\
\mathrm{mg} / \mathrm{l}\end{array}$ & $\begin{array}{c}\mathrm{Fe} \\
\mathrm{mg} / \mathrm{l}\end{array}$ & $\begin{array}{l}\mathrm{Mn} \\
\mathrm{mg} / \mathrm{l}\end{array}$ & $\begin{array}{c}\mathrm{Ni} \\
\mathrm{mg} / \mathrm{l}\end{array}$ & $\begin{array}{l}\mathrm{Pb} \\
\mathrm{mg} / \mathrm{l}\end{array}$ & $\begin{array}{c}\mathrm{Zn} \\
\mathrm{mg} / \mathrm{l}\end{array}$ & $\begin{array}{l}\mathrm{Tem} \\
\left({ }^{\circ} \mathrm{C}\right)\end{array}$ & $\begin{array}{c}\text { EC } \\
(\mu \mathrm{s} / \mathrm{cm})\end{array}$ & $\mathrm{pH}$ & $\begin{array}{l}\text { TDS } \\
(\mathrm{ppm})\end{array}$ \\
\hline Vwei & SW1 & Pond & 8.8811 & 9.7216 & $\begin{array}{l}+1 \\
0 \\
x \\
\text { in }\end{array}$ & $\begin{array}{l}\hat{b} \\
\stackrel{1}{x} \\
\text { an } \\
\text { in }\end{array}$ & 0 & $\begin{array}{l}\hat{o} \\
\stackrel{0}{x} \\
x \\
\ddot{n}\end{array}$ & $\begin{array}{l}\tilde{o} \\
\stackrel{x}{x} \\
\times \\
\underline{-} \\
-\end{array}$ & $\begin{array}{l}\vec{\nabla} \\
\stackrel{0}{i}\end{array}$ & $\begin{array}{l}\vec{b} \\
\stackrel{1}{x} \\
\stackrel{1}{-}\end{array}$ & $\begin{array}{l}\hat{m} \\
b \\
x \\
0 \\
\dot{m}\end{array}$ & $\begin{array}{l}\tilde{o} \\
\stackrel{0}{x} \\
\tilde{y} \\
\ddot{n}\end{array}$ & $\begin{array}{l}\hat{b} \\
\varrho \\
x \\
\hat{\imath} \\
0\end{array}$ & 23.6 & 880 & 8.5 & 563 \\
\hline Bisichi1 & SW2 & Pond & 8.8911 & 9.7158 & $\begin{array}{l}+1 \\
\stackrel{1}{0} \\
x \\
a\end{array}$ & $\begin{array}{l}0 \\
0 \\
x \\
n \\
\stackrel{1}{i}\end{array}$ & 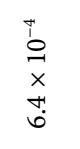 & $\begin{array}{l}3 \\
0 \\
x \\
x \\
\stackrel{n}{+}\end{array}$ & $\begin{array}{l}\hat{1} \\
0 \\
x \\
x \\
b \\
\dot{r}\end{array}$ & $\begin{array}{l}\infty \\
: \\
\stackrel{0}{0} \\
-i\end{array}$ & $\begin{array}{l}\vec{b} \\
\vec{x} \\
\times \\
\vec{\infty} \\
\stackrel{-}{-}\end{array}$ & $\begin{array}{l}0 \\
0 \\
x \\
\infty \\
\infty \\
a\end{array}$ & $\begin{array}{l}\tilde{b} \\
\vec{x} \\
\times \\
\tilde{b} \\
\dot{\lambda}\end{array}$ & $\begin{array}{l}\vec{b} \\
\stackrel{1}{x} \\
x \\
b \\
\infty \\
i\end{array}$ & 25.9 & 884 & 8.6 & 566 \\
\hline Bisichi2 & SW3 & Pond & 8.8947 & 9.7131 & 0 & 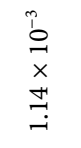 & 0 & $\begin{array}{l}\hat{b} \\
\stackrel{1}{x} \\
x \\
a \\
i\end{array}$ & $\begin{array}{l}0 \\
o \\
x \\
\tilde{a} \\
a\end{array}$ & $\begin{array}{l}\vec{b} \\
\stackrel{-}{x} \\
x \\
\stackrel{0}{0} \\
\dot{n}\end{array}$ & $\begin{array}{l}\hat{1} \\
0 \\
x \\
o \\
\vdots \\
\dot{m}\end{array}$ & 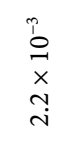 & $\begin{array}{l}n \\
0 \\
x \\
m \\
m \\
i n\end{array}$ & $\begin{array}{l}\tilde{\gamma} \\
\hat{\sigma} \\
\times \\
\hat{\sigma} \\
\dot{\lambda}\end{array}$ & 26.1 & 1024 & 8.5 & 655 \\
\hline Dunduma & SW4 & Pond & 8.9100 & 9.6936 & 0 & $\begin{array}{l}\hat{b} \\
\stackrel{1}{x} \\
\vec{\sim}\end{array}$ & $\begin{array}{l}0 \\
b \\
x \\
x\end{array}$ & $\begin{array}{l}\vec{b} \\
\stackrel{\vec{x}}{x} \\
\vec{i}\end{array}$ & $\begin{array}{l}\frac{0}{b} \\
\dot{x} \\
\overrightarrow{6}\end{array}$ & 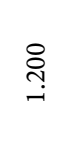 & 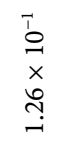 & $\begin{array}{l}\hat{b} \\
\stackrel{1}{x} \\
\times \\
\stackrel{i}{N}\end{array}$ & 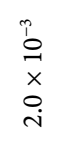 & $\begin{array}{l}\tilde{L} \\
\circ \\
x \\
\times \\
0 \\
\dot{m}\end{array}$ & 25.9 & 546 & 8.4 & 349 \\
\hline Zira & SW5 & Pond & 8.9053 & 9.7029 & 0 & $\begin{array}{l}+1 \\
\circ \\
x \\
x \\
\infty \\
\sigma\end{array}$ & 0 & $\begin{array}{l}\hat{b} \\
\stackrel{0}{1} \\
x \\
\infty \\
i\end{array}$ & $\begin{array}{l}\tilde{1} \\
0 \\
\times \\
\times \\
0 \\
0 \\
-\end{array}$ & $\begin{array}{l}\vec{b} \\
0 \\
x \\
\text { in } \\
\text { in }\end{array}$ & $\begin{array}{l}\hat{\tilde{O}} \\
\stackrel{1}{x} \\
\overrightarrow{\tilde{\sigma}}\end{array}$ & $\begin{array}{l}0 \\
0 \\
x \\
x \\
0 \\
i\end{array}$ & $\begin{array}{l}0 \\
0 \\
x \\
m \\
m \\
m\end{array}$ & 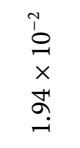 & 27.4 & 716 & 8.2 & 458 \\
\hline $\begin{array}{c}\text { Kuru } \\
\text { Jental }\end{array}$ & SW6 & Pond & 8.8825 & 9.6947 & 0 & $\begin{array}{l}\hat{b} \\
\stackrel{1}{x} \\
\hat{\sigma} \\
\stackrel{i}{i}\end{array}$ & $\begin{array}{l}0 \\
b \\
x \\
0 \\
0\end{array}$ & 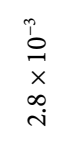 & $\begin{array}{l}\tilde{o} \\
\stackrel{1}{x} \\
x \\
\\
\end{array}$ & કे & $\begin{array}{l}\vec{b} \\
\vec{x} \\
x \\
\underline{n} \\
\vec{i}\end{array}$ & $\begin{array}{l}p \\
0 \\
x \\
0 \\
\dot{m}\end{array}$ & $\begin{array}{l}p \\
0 \\
\dot{x} \\
0 \\
\dot{b}\end{array}$ & $\begin{array}{l}\tilde{b} \\
\stackrel{1}{x} \\
\hat{\sigma} \\
\dot{0}\end{array}$ & 24.9 & 892 & 8.1 & 571 \\
\hline $\begin{array}{c}\text { Kuru } \\
\text { Jenta2 }\end{array}$ & SW7 & Pond & 8.8881 & 9.6966 & $\begin{array}{l}\overrightarrow{1} \\
\stackrel{1}{x} \\
\times \\
\infty\end{array}$ & 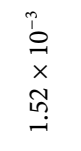 & $\begin{array}{l}+ \\
\dot{1} \\
\dot{x} \\
\infty \\
i \\
i\end{array}$ & 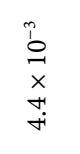 & $\begin{array}{l}\hat{1} \\
0 \\
x \\
i b \\
i \\
i\end{array}$ & $\begin{array}{l}\stackrel{n}{\Omega} \\
\stackrel{n}{n} \\
\dot{n}\end{array}$ & $\begin{array}{l}\vec{b} \\
\stackrel{1}{x} \\
\stackrel{-}{\sim}\end{array}$ & $\begin{array}{l}0 \\
b \\
\vec{x} \\
\dot{x} \\
\dot{b} \\
\text { in }\end{array}$ & $\begin{array}{l}\hat{r} \\
\stackrel{1}{x} \\
x \\
\tilde{n} \\
\stackrel{n}{-}\end{array}$ & 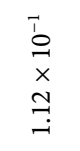 & 29.1 & 1856 & 8.1 & 1187 \\
\hline Rahai & SW8 & Pond & 8.9094 & 9.6869 & 0 & 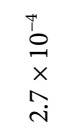 & 0 & $\begin{array}{l}\hat{b} \\
\stackrel{1}{x} \\
x \\
n \\
i\end{array}$ & $\begin{array}{l}\hat{b} \\
0 \\
x \\
\infty \\
0 \\
b\end{array}$ & $\begin{array}{l}\tilde{1} \\
0 \\
\times \\
x \\
+1 \\
\infty\end{array}$ & $\begin{array}{l}\tilde{b} \\
\stackrel{1}{x} \\
\tilde{\sigma} \\
\stackrel{\sigma}{-}\end{array}$ & $\begin{array}{l}p \\
0 \\
x \\
x \\
\infty \\
-\end{array}$ & $\begin{array}{l}0 \\
0 \\
\vec{x} \\
\times \\
0 \\
-\end{array}$ & $\begin{array}{l}\tilde{b} \\
\stackrel{1}{x} \\
\stackrel{+}{m} \\
\stackrel{-}{+}\end{array}$ & 28.0 & 932 & 8.2 & 597 \\
\hline Rassa1 & SW9 & Pond & 8.9272 & 9.6951 & $\begin{array}{l}m \\
\stackrel{b}{1} \\
x \\
m \\
-\end{array}$ & $\begin{array}{l}\hat{L} \\
\stackrel{1}{x} \\
x \\
\stackrel{n}{n}\end{array}$ & 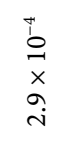 & 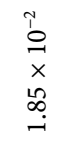 & $\begin{array}{l}\hat{b} \\
\stackrel{-}{x} \\
x \\
\hat{n} \\
\dot{H}\end{array}$ & $\begin{array}{l}\vec{b} \\
\stackrel{\vec{x}}{x} \\
\stackrel{x}{o} \\
\stackrel{i}{i}\end{array}$ & $\begin{array}{l}n \\
\delta \\
\infty \\
\infty \\
i \\
i\end{array}$ & $\begin{array}{l}\tilde{1} \\
\stackrel{1}{x} \\
x \\
o \\
\stackrel{1}{-}\end{array}$ & $\begin{array}{l}\tilde{o} \\
\stackrel{1}{x} \\
x \\
\tilde{N} \\
\infty \\
\infty\end{array}$ & $\begin{array}{l}\hat{\delta} \\
\stackrel{1}{x} \\
x \\
\tilde{O} \\
\sigma\end{array}$ & 26.8 & 1596 & 8.4 & 1021 \\
\hline Rassa 2 & SW10 & Pond & 8.9294 & 9.6973 & 0 & $\begin{array}{l}\tilde{b} \\
\stackrel{1}{1} \\
x \\
\infty \\
b \\
\infty \\
\infty\end{array}$ & $\begin{array}{l}\text { n } \\
\stackrel{1}{1} \\
x \\
\text { in }\end{array}$ & $\begin{array}{l}\hat{b} \\
\stackrel{0}{x} \\
\stackrel{n}{n}\end{array}$ & $\begin{array}{l}\tilde{b} \\
\stackrel{1}{x} \\
\times \\
\tilde{N} \\
\stackrel{-}{-}\end{array}$ & $\underset{m}{m}$ & $\begin{array}{l}\vec{b} \\
\vec{x} \\
\vec{\sigma} \\
\dot{n}\end{array}$ & $\begin{array}{l}\stackrel{p}{0} \\
\stackrel{1}{x} \\
\underset{+}{+}\end{array}$ & 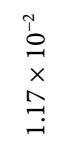 & $\begin{array}{l}\tilde{b} \\
\hat{O} \\
\times \\
\tilde{V} \\
\dot{r}\end{array}$ & 29.5 & 876 & 8.5 & 561 \\
\hline Gassa1 & SW11 & Pond & 8.8956 & 9.5741 & 0 & $\begin{array}{l}\hat{b} \\
\stackrel{0}{x} \\
\vec{b} \\
\stackrel{i}{1}\end{array}$ & $\begin{array}{l}\text { ì } \\
\stackrel{1}{1} \\
x \\
\stackrel{-}{-}\end{array}$ & $\begin{array}{l}\hat{b} \\
\stackrel{\vec{\gamma}}{x} \\
\dot{x} \\
\underset{+}{+}\end{array}$ & $\begin{array}{l}\hat{o} \\
\stackrel{1}{x} \\
\dot{x} \\
\stackrel{n}{-}\end{array}$ & $\begin{array}{l}n \\
\infty \\
\infty \\
-1\end{array}$ & $\begin{array}{l}\vec{b} \\
\stackrel{x}{x} \\
\vec{n} \\
-\end{array}$ & $\begin{array}{l}\hat{b} \\
0 \\
x \\
b \\
\dot{n}\end{array}$ & $\begin{array}{l}0 \\
o \\
\vec{x} \\
m \\
\infty\end{array}$ & $\begin{array}{l}\tilde{c} \\
\vec{x} \\
x \\
m \\
\infty \\
\infty\end{array}$ & 29.5 & 264 & 8.8 & 169 \\
\hline Gassa2 & SW12 & Pond & 8.8969 & 9.5742 & 0 & $\begin{array}{l}\text { to } \\
\circ \\
\dot{x} \\
o \\
\sigma\end{array}$ & 0 & $\begin{array}{l}\hat{b} \\
\stackrel{1}{x} \\
x \\
b \\
i\end{array}$ & $\begin{array}{l}p \\
b \\
x \\
x \\
n \\
+ \\
+\end{array}$ & $\begin{array}{l}\vec{b} \\
\stackrel{0}{1} \\
x \\
\stackrel{x}{+}\end{array}$ & $\begin{array}{l}\tilde{b} \\
0 \\
x \\
\llcorner \\
\stackrel{1}{r}\end{array}$ & $\begin{array}{l}0 \\
0 \\
x \\
\\
\end{array}$ & $\begin{array}{l}0 \\
0 \\
\stackrel{0}{x} \\
\stackrel{+}{-} \\
-\end{array}$ & $\begin{array}{l}\tilde{o} \\
\stackrel{-}{x} \\
\times \\
\tilde{m} \\
\stackrel{-}{-}\end{array}$ & 27.6 & 486 & 8.6 & 311 \\
\hline Britvic & SW13 & Pond & 8.9078 & 9.5638 & 0 & 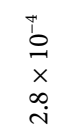 & 0 & $\begin{array}{l}\hat{b} \\
\stackrel{1}{x} \\
\dot{x} \\
\overrightarrow{-}\end{array}$ & $\begin{array}{l}\dot{b} \\
b \\
x \\
\dot{x} \\
\dot{1}\end{array}$ & $\begin{array}{l}\vec{b} \\
0 \\
x \\
\overrightarrow{0} \\
\dot{i}\end{array}$ & $\begin{array}{l}\hat{1} \\
0 \\
x \\
0 \\
\dot{r}\end{array}$ & $\begin{array}{l}\mathfrak{m} \\
\stackrel{0}{x} \\
x \\
m \\
-\end{array}$ & $\begin{array}{l}\hat{b} \\
\stackrel{1}{x} \\
\dot{x} \\
\stackrel{-}{-}\end{array}$ & 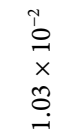 & 28.2 & 300 & 8.4 & 193 \\
\hline
\end{tabular}




\section{Continued}

\begin{tabular}{|c|c|c|c|c|c|c|c|c|c|c|c|c|c|c|c|c|c|c|}
\hline $\begin{array}{l}\text { Hwol } \\
\text { Gassa }\end{array}$ & SW14 & Pond & 8.9097 & 9.5634 & 0 & $\begin{array}{l}0 \\
0 \\
x \\
o \\
\stackrel{0}{-}\end{array}$ & $\begin{array}{l}\hat{b} \\
\stackrel{0}{x} \\
\hat{x}\end{array}$ & $\begin{array}{l}\hat{b} \\
\stackrel{0}{x} \\
\hat{i}\end{array}$ & $\begin{array}{l}\hat{\jmath} \\
\stackrel{0}{x} \\
\times \\
\stackrel{-}{0}\end{array}$ & $\begin{array}{l}\vec{b} \\
\stackrel{0}{1} \\
x \\
\stackrel{+}{+} \\
\stackrel{1}{n}\end{array}$ & $\begin{array}{l}\hat{1} \\
\circ \\
x \\
\text { x } \\
\infty \\
\text { in }\end{array}$ & 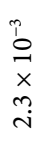 & $\begin{array}{l}\hat{b} \\
\stackrel{1}{x} \\
x \\
b \\
i\end{array}$ & $\begin{array}{l}\tilde{u} \\
0 \\
\times \\
\infty \\
\infty \\
\dot{m}\end{array}$ & 26 & 1620 & 7.6 & 1037 \\
\hline $\begin{array}{c}\text { Pol.BarrK } \\
\text { B/Ladi }\end{array}$ & SW15 & Pond & 8.8956 & 9.5515 & 0 & $\begin{array}{l}\overrightarrow{1} \\
0 \\
x \\
x \\
0 \\
i\end{array}$ & 0 & $\begin{array}{l}\hat{b} \\
\stackrel{0}{x} \\
\vec{i} \\
\vec{i}\end{array}$ & $\begin{array}{l}m \\
b \\
x \\
n \\
0 \\
0\end{array}$ & $\begin{array}{l}\vec{b} \\
\stackrel{0}{1} \\
x \\
n \\
\stackrel{0}{0}\end{array}$ & $\begin{array}{l}\tilde{b} \\
ٍ \\
x \\
\hat{x} \\
\hat{n}\end{array}$ & 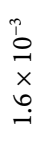 & $\begin{array}{l}\hat{b} \\
\stackrel{0}{x} \\
\stackrel{+}{-} \\
-\end{array}$ & 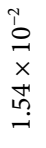 & 28.7 & 444 & 8.6 & 284 \\
\hline Rakung & SW16 & Pond & 8.8850 & 9.5411 & 0 & $\begin{array}{c}0 \\
0 \\
x \\
x \\
\tilde{N} \\
\infty \\
i\end{array}$ & $\begin{array}{l}b \\
\stackrel{0}{1} \\
x \\
a\end{array}$ & $\begin{array}{l}\tilde{b} \\
\stackrel{0}{x} \\
x \\
\dot{n} \\
\dot{m}\end{array}$ & $\begin{array}{l}\tilde{\imath} \\
\stackrel{0}{x} \\
x \\
\tilde{a} \\
\end{array}$ & 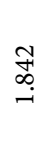 & $\begin{array}{l}\vec{b} \\
\stackrel{\overrightarrow{1}}{x} \\
\times \\
\stackrel{-}{-}\end{array}$ & $\begin{array}{l}\hat{b} \\
\stackrel{1}{x} \\
\vec{m}\end{array}$ & $\begin{array}{l}\tilde{b} \\
\stackrel{1}{x} \\
\stackrel{-}{-}\end{array}$ & $\begin{array}{l}\tilde{L} \\
\stackrel{1}{x} \\
\times \\
\infty \\
\stackrel{+}{+} \\
+\end{array}$ & 28.2 & 334 & 8.4 & 214 \\
\hline Kworos1 & SW17 & Pond & 8.9008 & 9.5409 & 0 & $\begin{array}{l}+1 \\
0 \\
x \\
x \\
+ \\
\infty\end{array}$ & 0 & $\begin{array}{l}\hat{b} \\
\stackrel{0}{1} \\
\times \\
\hat{a} \\
\vec{i}\end{array}$ & $\begin{array}{l}0 \\
b \\
x \\
\vec{n}\end{array}$ & ڤิ & 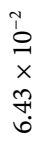 & $\begin{array}{l}m \\
\stackrel{b}{1} \\
x \\
m \\
i\end{array}$ & $\begin{array}{l}0 \\
\stackrel{1}{0} \\
\times \\
g \\
\dot{+}\end{array}$ & 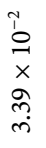 & 27.2 & 1444 & 8.5 & 924 \\
\hline Kworos2 & SW18 & Pond & 8.9022 & 9.5440 & 0 & $\begin{array}{l}\vec{b} \\
\stackrel{0}{x} \\
\vec{x} \\
\vec{i}\end{array}$ & 0 & $\begin{array}{l}\hat{b} \\
\stackrel{0}{x} \\
\vec{m}\end{array}$ & $\begin{array}{l}0 \\
\stackrel{0}{1} \\
x \\
\stackrel{n}{i n}\end{array}$ & $\begin{array}{l}\vec{b} \\
\stackrel{0}{1} \\
x \\
\overrightarrow{0} \\
\dot{n}\end{array}$ & $\begin{array}{l}\tilde{b} \\
\varrho \\
x \\
b \\
+1 \\
\dot{+}\end{array}$ & $\begin{array}{l}p \\
0 \\
x \\
\stackrel{1}{0} \\
i\end{array}$ & 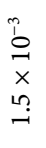 & $\begin{array}{l}\tilde{o} \\
\stackrel{0}{x} \\
x \\
\stackrel{n}{-}\end{array}$ & 28.4 & 852 & 8.7 & 341 \\
\hline Kworos3 & SW19 & Pond & 8.8994 & 9.5535 & 0 & 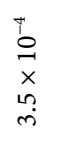 & 0 & $\begin{array}{l}\hat{b} \\
\stackrel{0}{x} \\
a \\
\hat{i}\end{array}$ & $\begin{array}{l}\hat{b} \\
\stackrel{0}{x} \\
\stackrel{1}{n}\end{array}$ & $\begin{array}{l}\vec{b} \\
\stackrel{1}{1} \\
\times \\
\stackrel{q}{+} \\
i\end{array}$ & 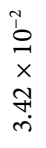 & $\begin{array}{l}0 \\
0 \\
x \\
\infty \\
\infty \\
-\end{array}$ & $\begin{array}{l}0 \\
0 \\
x \\
\\
-i\end{array}$ & $\begin{array}{l}\tilde{o} \\
\stackrel{0}{x} \\
\vec{\sigma} \\
\stackrel{-}{-}\end{array}$ & 28.2 & 600 & 8.4 & 384 \\
\hline Pwamol1 & SW20 & Stream & 8.8881 & 9.6555 & 0 & $\begin{array}{l}\overrightarrow{1} \\
0 \\
x \\
\overrightarrow{0}\end{array}$ & 0 & $\begin{array}{l}\hat{b} \\
\stackrel{1}{x} \\
x \\
0 \\
\dot{+}\end{array}$ & 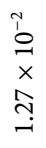 & $\underset{\stackrel{\infty}{+}}{\stackrel{\infty}{m}}$ & $\begin{array}{l}\tilde{b} \\
0 \\
x \\
\hat{A} \\
i n\end{array}$ & $\begin{array}{l}\tilde{b} \\
\stackrel{0}{1} \\
\times \\
m \\
\dot{r}\end{array}$ & 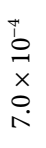 & 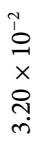 & 26.8 & 182 & 7.8 & 117 \\
\hline Pwamol2 & SW21 & Stream & 8.8955 & 9.6515 & 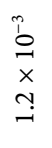 & $\begin{array}{l}\vec{y} \\
\circ \\
\dot{x} \\
\dot{0} \\
\dot{a}\end{array}$ & 0 & $\begin{array}{l}\hat{b} \\
\stackrel{1}{x} \\
x \\
0 \\
\dot{+}\end{array}$ & $\begin{array}{l}\mathfrak{b} \\
\stackrel{0}{x} \\
x \\
\stackrel{y}{y}\end{array}$ & $\begin{array}{l}\vec{b} \\
\stackrel{0}{x} \\
x \\
ت \\
ت\end{array}$ & $\begin{array}{l}\vec{b} \\
\stackrel{1}{x} \\
\times \\
\sim \\
\stackrel{n}{n}\end{array}$ & $\begin{array}{l}\hat{b} \\
\stackrel{1}{x} \\
x \\
\hat{i}\end{array}$ & 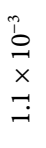 & $\begin{array}{l}\tilde{y} \\
0 \\
x \\
\hat{n} \\
\end{array}$ & 22.8 & 1580 & 7.2 & 1011 \\
\hline Chit & SW22 & Stream & 8.8992 & 9.6376 & 0 & $\begin{array}{l}\hat{b} \\
0 \\
x \\
\dot{b} \\
\dot{0}\end{array}$ & 0 & $\begin{array}{l}m \\
\stackrel{0}{1} \\
x \\
m \\
m\end{array}$ & 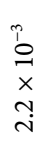 & $\stackrel{\widehat{N}}{\text { i }}$ & $\begin{array}{l}\hat{1} \\
\vec{x} \\
x \\
o \\
0 \\
0\end{array}$ & $\begin{array}{l}\hat{b} \\
0 \\
x \\
\mathfrak{d} \\
i\end{array}$ & 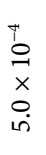 & 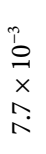 & 26.1 & 210 & 7.7 & 135 \\
\hline Kassa & SW23 & Stream & 8.9029 & 9.5974 & 0 & $\begin{array}{l}\ddot{1} \\
\stackrel{0}{0} \\
\times \\
0 \\
0\end{array}$ & 0 & $\begin{array}{l}\hat{b} \\
\stackrel{0}{x} \\
\vec{m}\end{array}$ & $\begin{array}{l}\hat{b} \\
\stackrel{0}{x} \\
x \\
\vec{t} \\
\dot{n}\end{array}$ & $\begin{array}{l}\stackrel{9}{+} \\
\dot{m} \\
\dot{m}\end{array}$ & $\begin{array}{l}\vec{b} \\
\stackrel{\vec{x}}{x} \\
\vec{\exists} \\
\overrightarrow{+}\end{array}$ & $\begin{array}{l}\hat{b} \\
\stackrel{0}{1} \\
x \\
\infty \\
\dot{i}\end{array}$ & 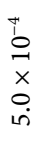 & $\begin{array}{l}\tilde{y} \\
\stackrel{0}{x} \\
x \\
\tilde{N} \\
\end{array}$ & 27.5 & 304 & 7.8 & 195 \\
\hline \multicolumn{5}{|c|}{ Nigerian Standard Guideline Value } & $\begin{array}{l}\underset{0}{0} \\
\underset{x}{-}\end{array}$ & & $\begin{array}{l}\hat{p} \\
\stackrel{0}{1} \\
x \\
m\end{array}$ & $\begin{array}{l}\hat{1} \\
\stackrel{1}{1} \\
\times \\
0 \\
\text { in }\end{array}$ & $\neg$ & $\begin{array}{l}\vec{r} \\
\circ \\
\dot{x} \\
\dot{0} \\
\dot{r}\end{array}$ & $\begin{array}{l}\vec{b} \\
\circ \\
\times \\
\stackrel{x}{0} \\
\dot{i}\end{array}$ & $\begin{array}{l}\tilde{i} \\
\stackrel{1}{ } \\
\times \\
0 \\
\dot{i}\end{array}$ & $\begin{array}{l}\tilde{1} \\
\stackrel{1}{0} \\
\times \\
\circ \\
-\end{array}$ & m & & & $6.5-8.5$ & $\times 10^{-2}$ \\
\hline \multicolumn{5}{|c|}{ Minimum } & 0 & $\begin{array}{l}\vec{y} \\
\stackrel{0}{x} \\
\times \\
\vec{i}\end{array}$ & 0 & $\begin{array}{l}\hat{b} \\
\stackrel{0}{x} \\
\times \\
\stackrel{a}{-}\end{array}$ & $\begin{array}{l}0 \\
b \\
x \\
n \\
+ \\
+\end{array}$ & $\begin{array}{l}\hat{T} \\
\stackrel{1}{1} \\
\times \\
+\underset{\infty}{\infty}\end{array}$ & 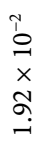 & $\begin{array}{l}\mathfrak{p} \\
\stackrel{0}{0} \\
\times \\
\mathfrak{m}\end{array}$ & 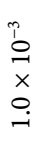 & $\begin{array}{l}\tilde{1} \\
0 \\
x \\
\tilde{x} \\
\stackrel{-}{-}\end{array}$ & 22.8 & 182 & 7.2 & $\times 10^{-2}$ \\
\hline \multicolumn{5}{|c|}{ Maximum } & $\begin{array}{l}\hat{b} \\
\stackrel{\vec{x}}{x} \\
\stackrel{m}{-}\end{array}$ & $\begin{array}{l}\tilde{o} \\
0 \\
x \\
\dot{x} \\
\stackrel{+}{n}\end{array}$ & \begin{tabular}{l}
$\ddot{H}$ \\
$\stackrel{0}{1}$ \\
$x$ \\
\multirow{b}{0}{}
\end{tabular} & $\begin{array}{l}\hat{\tilde{O}} \\
\stackrel{1}{x} \\
x \\
0 \\
\infty \\
-\end{array}$ & 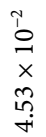 & $\begin{array}{l}\vec{b} \\
0 \\
x \\
x \\
\tilde{\sigma} \\
\dot{i}\end{array}$ & $\begin{array}{l}n \\
\mathbb{0} \\
\infty \\
i \\
i\end{array}$ & 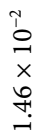 & $\begin{array}{l}\tilde{b} \\
0 \\
x \\
\underset{1}{x} \\
\hat{n} \\
\infty\end{array}$ & $\begin{array}{l}\vec{b} \\
0 \\
x \\
\dot{x} \\
\infty \\
\infty \\
i\end{array}$ & 29.5 & 1856 & 8.8 & $\times 10^{-2}$ \\
\hline
\end{tabular}




\begin{tabular}{|c|c|c|c|c|c|c|c|c|c|c|c|c|c|c|}
\hline Mean & $\begin{array}{l}\vec{t} \\
\stackrel{1}{x} \\
\underset{\sim}{x} \\
\sim\end{array}$ & $\begin{array}{l}\hat{b} \\
\qquad \\
x \\
\& \\
\dot{b}\end{array}$ & 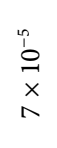 & $\begin{array}{l}\hat{b} \\
\dot{o} \\
\dot{x} \\
\stackrel{a}{m}\end{array}$ & $\begin{array}{l}\hat{\sigma} \\
\hat{\sigma} \\
x \\
\stackrel{m}{-}\end{array}$ & $\begin{array}{l}\text { ¿゙ } \\
\text { in } \\
\text { in }\end{array}$ & $\begin{array}{l}\vec{b} \\
\hat{0} \\
\times \\
a \\
\stackrel{a}{i}\end{array}$ & $\begin{array}{l}0 \\
\hat{O} \\
x \\
\dot{x} \\
\dot{m}\end{array}$ & $\begin{array}{l}\tilde{\imath} \\
\stackrel{0}{x} \\
x \\
\tilde{n} \\
\end{array}$ & $\begin{array}{l}\tilde{0} \\
0 \\
x \\
\dot{0} \\
\dot{0}\end{array}$ & 27.1 & 818.4 & 8.2 & $\times 10^{-2}$ \\
\hline Standard deviation & $\begin{array}{l}\ddot{0} \\
\stackrel{0}{x} \\
x \\
\forall\end{array}$ & $\begin{array}{l}\tilde{r} \\
\dot{0} \\
x \\
\end{array}$ & 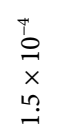 & $\begin{array}{l}\hat{b} \\
0 \\
x \\
\dot{x} \\
\dot{n}\end{array}$ & $\begin{array}{l}\tilde{0} \\
0 \\
\dot{x} \\
0 \\
0 \\
\end{array}$ & $\underset{+}{\stackrel{8}{+}}$ & $\begin{array}{l}\vec{b} \\
\vec{x} \\
\vec{A} \\
\vec{n}\end{array}$ & $\begin{array}{l}p \\
b \\
x \\
x \\
\dot{n}\end{array}$ & $\begin{array}{l}\tilde{r} \\
o \\
x \\
\vec{x} \\
\tilde{N}\end{array}$ & $\begin{array}{l}\tilde{r} \\
0 \\
x \\
\dot{x} \\
\tilde{r}\end{array}$ & 1.74 & 504.6 & 0.4 & $\times 10^{-2}$ \\
\hline
\end{tabular}

Table 5. Heavy metal concentration and physical parameters in groundwater.

\begin{tabular}{|c|c|c|c|c|c|c|c|c|c|c|c|c|c|c|c|c|c|c|}
\hline $\begin{array}{l}\text { Sample } \\
\text { location }\end{array}$ & $\begin{array}{c}\text { Sample } \\
\text { No }\end{array}$ & Des & Long & Lat & $\begin{array}{c}\text { As } \\
\mathrm{mg} / \mathrm{l}\end{array}$ & $\begin{array}{c}\text { Co } \\
\mathrm{mg} / \mathrm{l}\end{array}$ & $\begin{array}{c}\mathrm{Cd} \\
\mathrm{mg} / \mathrm{l}\end{array}$ & $\begin{array}{c}\mathrm{Cr} \\
\mathrm{mg} / \mathrm{l}\end{array}$ & $\begin{array}{c}\mathrm{Cu} \\
\mathrm{mg} / \mathrm{l}\end{array}$ & $\begin{array}{c}\mathrm{Fe} \\
\mathrm{mg} / \mathrm{l}\end{array}$ & $\begin{array}{c}\mathrm{Mn} \\
\mathrm{mg} / \mathrm{l}\end{array}$ & $\begin{array}{c}\mathrm{Ni} \\
\mathrm{mg} / \mathrm{l}\end{array}$ & $\begin{array}{c}\mathrm{Pb} \\
\mathrm{mg} / \mathrm{l}\end{array}$ & $\begin{array}{c}\mathrm{Zn} \\
\mathrm{mg} / \mathrm{l}\end{array}$ & $\begin{array}{l}\text { Tem } \\
\left({ }^{\circ} \mathrm{C}\right)\end{array}$ & $\begin{array}{c}\mathrm{EC} \\
(\mu \mathrm{s} / \mathrm{cm})\end{array}$ & $\mathrm{pH}$ & $\begin{array}{c}\text { TDS } \\
(\mathrm{ppm})\end{array}$ \\
\hline Vweil & GW1 & HDW & 8.8775 & 9.7231 & 0 & $\begin{array}{l}\hat{r} \\
\stackrel{0}{1} \\
\times \\
0 \\
-\end{array}$ & 0 & $\begin{array}{l}\hat{b} \\
\stackrel{0}{x} \\
\dot{x} \\
\hat{i}\end{array}$ & 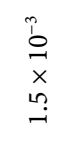 & $\begin{array}{l}\vec{b} \\
\stackrel{1}{x} \\
x \\
\stackrel{m}{m}\end{array}$ & $\begin{array}{l}\tilde{b} \\
0 \\
\times \\
\hat{\sigma} \\
\dot{m}\end{array}$ & $\begin{array}{l}\hat{b} \\
\stackrel{1}{x} \\
\times \\
\stackrel{\infty}{-}\end{array}$ & $\begin{array}{l}p \\
b \\
\times \\
ت \\
-1\end{array}$ & 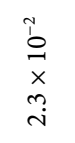 & 23.3 & 365 & 6.5 & 168 \\
\hline Vwei2 & GW2 & HDW & 8.8734 & 9.7216 & 0 & 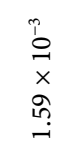 & 0 & $\begin{array}{l}\hat{b} \\
0 \\
\times \\
\stackrel{n}{m}\end{array}$ & 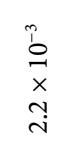 & $\begin{array}{l}\vec{b} \\
\stackrel{1}{x} \\
x \\
\infty \\
\vec{i}\end{array}$ & 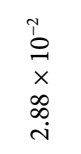 & 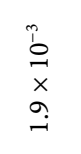 & $\begin{array}{l}\mathfrak{b} \\
\stackrel{1}{1} \\
x \\
\stackrel{-}{-}\end{array}$ & $\begin{array}{l}i \\
0 \\
x \\
\text { x } \\
\stackrel{2}{-}\end{array}$ & 23.6 & 160 & 6.6 & 102 \\
\hline Bisichil & GW3 & HDW & 8.9034 & 9.7159 & 0 & $\begin{array}{l}\hat{b} \\
\stackrel{1}{1} \\
x \\
\infty \\
\stackrel{\sim}{-}\end{array}$ & 0 & $\begin{array}{l}\tilde{\delta} \\
\stackrel{1}{x} \\
x \\
\tilde{N} \\
\end{array}$ & $\begin{array}{l}\hat{m} \\
b \\
\dot{x} \\
\stackrel{+}{-}\end{array}$ & $\begin{array}{l}\vec{b} \\
0 \\
x \\
0 \\
\stackrel{1}{i} \\
\end{array}$ & 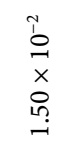 & $\begin{array}{l}\hat{m} \\
b \\
x \\
\stackrel{1}{n}\end{array}$ & 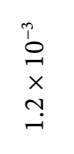 & 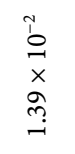 & 25.3 & 345 & 6.4 & 221 \\
\hline Bisichi 2 & GW4 & HDW & 8.9081 & 9.7200 & 0 & $\begin{array}{l}+1 \\
0 \\
\dot{1} \\
\dot{x} \\
0 \\
\dot{0}\end{array}$ & 0 & $\begin{array}{l}\hat{b} \\
\stackrel{1}{1} \\
x \\
\stackrel{a}{a} \\
a\end{array}$ & $\begin{array}{l}\dot{m} \\
0 \\
x \\
-1 \\
-1\end{array}$ & $\begin{array}{l}\hat{i} \\
\stackrel{1}{1} \\
x \\
\infty \\
\stackrel{\infty}{\wedge}\end{array}$ & $\begin{array}{l}\hat{b} \\
\dot{x} \\
\dot{0} \\
\dot{0}\end{array}$ & $\begin{array}{l}0 \\
0 \\
x \\
\stackrel{n}{x} \\
\stackrel{n}{-}\end{array}$ & $\begin{array}{l}\tilde{b} \\
0 \\
x \\
m \\
m\end{array}$ & $\begin{array}{l}\vec{b} \\
\stackrel{1}{x} \\
x \\
\text { aे } \\
\dot{+}\end{array}$ & 26.1 & 286 & 6.3 & 183 \\
\hline Bisichi3 & GW5 & HDW & 8.9096 & 9.7134 & 0 & 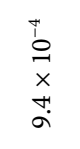 & 0 & 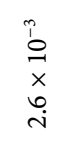 & $\begin{array}{l}\vec{b} \\
\stackrel{1}{x} \\
x \\
\vec{i}\end{array}$ & $\begin{array}{l}\vec{b} \\
0 \\
x \\
a \\
a \\
i\end{array}$ & $\begin{array}{l}\hat{r} \\
0 \\
x \\
n \\
0 \\
\dot{n}\end{array}$ & $\begin{array}{l}\vec{b} \\
\stackrel{1}{x} \\
\dot{x} \\
0 \\
\dot{i}\end{array}$ & 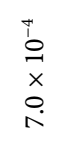 & $\begin{array}{l}\hat{\imath} \\
\stackrel{1}{x} \\
\times \\
\infty \\
\infty \\
-\end{array}$ & 26.3 & 209 & 6.5 & 134 \\
\hline Rassal & GW6 & HDW & 8.9360 & 9.6974 & 0 & $\begin{array}{l}p \\
0 \\
\times \\
\text { × } \\
\stackrel{q}{-}\end{array}$ & 0 & $\begin{array}{l}\hat{b} \\
\stackrel{0}{1} \\
x \\
n \\
i \\
i\end{array}$ & $\begin{array}{l}\dot{m} \\
b \\
\dot{x} \\
\dot{0} \\
-\end{array}$ & $\begin{array}{l}\tilde{r} \\
\stackrel{1}{x} \\
x \\
+! \\
\dot{b}\end{array}$ & $\begin{array}{l}\tilde{b} \\
\stackrel{1}{x} \\
\stackrel{\alpha}{\alpha} \\
\dot{+}\end{array}$ & $\begin{array}{l}\hat{b} \\
\stackrel{0}{1} \\
x \\
\stackrel{x}{-}\end{array}$ & 0 & $\begin{array}{l}\tilde{L} \\
\stackrel{1}{1} \\
\times \\
\dot{0} \\
\dot{i}\end{array}$ & 31.9 & 412 & 6.0 & 264 \\
\hline Rassa2 & GW7 & $\mathrm{BH}$ & 8.9285 & 9.6973 & 0 & $\begin{array}{l}+1 \\
0 \\
\vec{x} \\
\text { in } \\
\stackrel{+}{+}\end{array}$ & 0 & $\begin{array}{l}\vec{b} \\
\stackrel{1}{1} \\
\times \\
\vec{i}\end{array}$ & $\begin{array}{l}p \\
\stackrel{0}{0} \\
x \\
\stackrel{-}{-}\end{array}$ & $\begin{array}{l}\vec{b} \\
\stackrel{-}{1} \\
x \\
\vec{\infty} \\
\stackrel{\infty}{-}\end{array}$ & 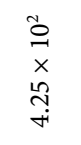 & $\begin{array}{l}\stackrel{3}{1} \\
\times \\
n \\
-1\end{array}$ & $\begin{array}{l}\text { 苍 } \\
\stackrel{1}{x} \\
0 \\
\text { in }\end{array}$ & $\begin{array}{l}\tilde{O} \\
\stackrel{0}{x} \\
\stackrel{0}{\hat{n}} \\
\stackrel{+}{+}\end{array}$ & 26.5 & 290 & 6.6 & 186 \\
\hline Rassa3 & GW8 & $\mathrm{BH}$ & 8.9310 & 9.7071 & 0 & $\begin{array}{l}\vec{t} \\
\stackrel{1}{1} \\
x \\
m \\
\tilde{b}\end{array}$ & 0 & $\begin{array}{l}\vec{b} \\
\stackrel{1}{x} \\
x \\
v \\
\tilde{b}\end{array}$ & $\begin{array}{l}\tilde{b} \\
\stackrel{1}{x} \\
\vec{\sim} \\
\stackrel{n}{x}\end{array}$ & $\begin{array}{l}\vec{b} \\
\vec{x} \\
\overrightarrow{+} \\
\vec{r}\end{array}$ & $\begin{array}{l}\tilde{b} \\
\stackrel{1}{x} \\
m \\
\stackrel{m}{-}\end{array}$ & $\begin{array}{l}\dot{b} \\
\stackrel{1}{1} \\
\dot{x} \\
\infty \\
\dot{m}\end{array}$ & $\begin{array}{l}\dot{p} \\
0 \\
x \\
n \\
n \\
m\end{array}$ & $\begin{array}{l}\hat{b} \\
\hat{x} \\
x \\
\hat{a} \\
\hat{\sigma}\end{array}$ & 26.2 & 220 & 6.0 & 141 \\
\hline Dunduma & GW9 & HDW & 8.9099 & 9.6881 & 0 & $\begin{array}{l}+1 \\
\dot{1} \\
x \\
\stackrel{x}{\hat{n}}\end{array}$ & 0 & $\begin{array}{l}\vec{b} \\
\overrightarrow{0} \\
\dot{x} \\
\vec{i} \\
\dot{i}\end{array}$ & $\begin{array}{l}\dot{m} \\
b \\
\dot{x} \\
\dot{b} \\
\dot{m}\end{array}$ & $\begin{array}{l}\vec{b} \\
\stackrel{1}{x} \\
x \\
\vec{\infty} \\
a \\
\sigma\end{array}$ & $\begin{array}{l}\stackrel{\tilde{o}}{\circ} \\
\underset{x}{x} \\
\stackrel{m}{-}\end{array}$ & $\begin{array}{l}\hat{m} \\
\stackrel{0}{1} \\
\times \\
0 \\
i\end{array}$ & $\begin{array}{l}\overrightarrow{1} \\
0 \\
\dot{x} \\
\dot{0} \\
0 \\
0\end{array}$ & $\begin{array}{l}\tilde{o} \\
\stackrel{1}{x} \\
x \\
\tilde{\sigma} \\
\stackrel{-}{-}\end{array}$ & 24.6 & 291 & 6.4 & 186 \\
\hline Dalor & GW10 & $\mathrm{BH}$ & 8.8977 & 9.6888 & 0 & $\begin{array}{l}\vec{t} \\
0 \\
\vec{x} \\
\stackrel{\sim}{r}\end{array}$ & 0 & $\begin{array}{l}p \\
0 \\
x \\
x \\
\stackrel{n}{r}\end{array}$ & 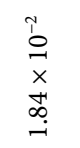 & $\begin{array}{l}\vec{b} \\
b \\
x \\
0 \\
\stackrel{0}{d} \\
\dot{v}\end{array}$ & 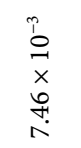 & $\begin{array}{l}\hat{b} \\
\stackrel{1}{x} \\
x \\
\stackrel{-}{r}\end{array}$ & $\begin{array}{l}\tilde{b} \\
\stackrel{0}{1} \\
x \\
m \\
-\end{array}$ & $\begin{array}{l}i \\
0 \\
x \\
\dot{0} \\
\vdots \\
\stackrel{1}{d}\end{array}$ & 26.5 & 772 & 6.2 & 494 \\
\hline
\end{tabular}




\section{Continued}

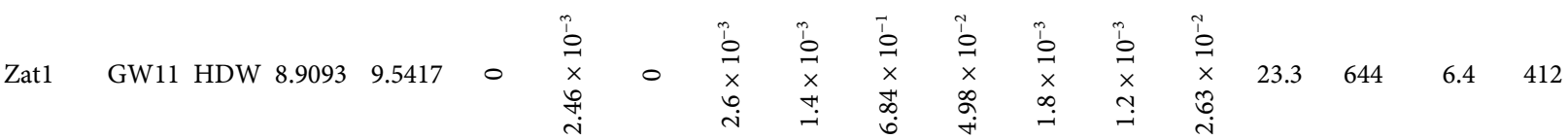

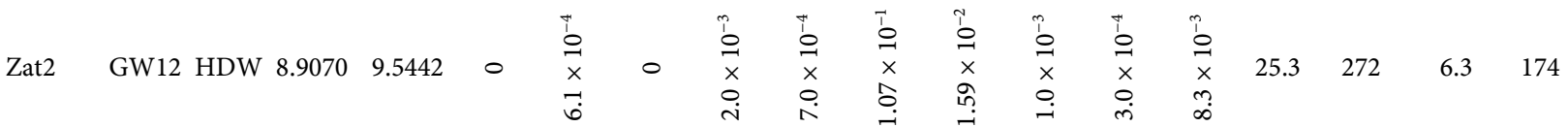

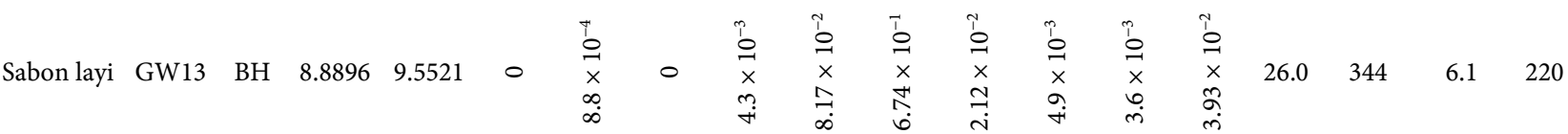

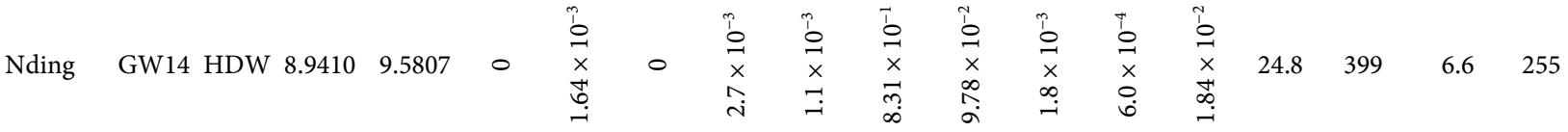

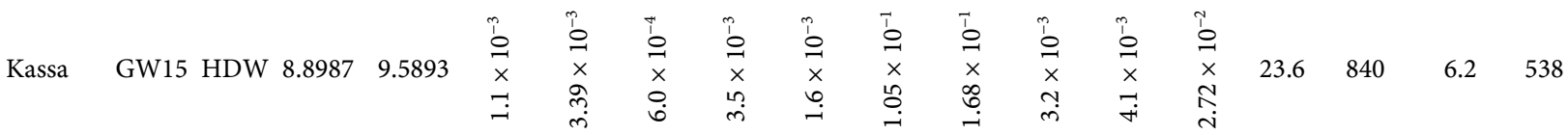

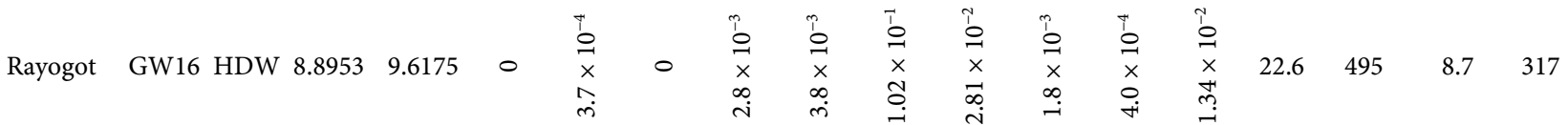

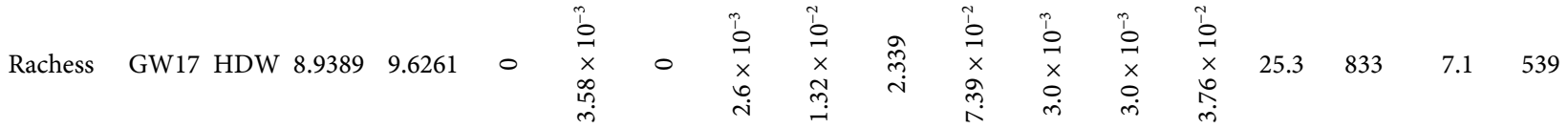

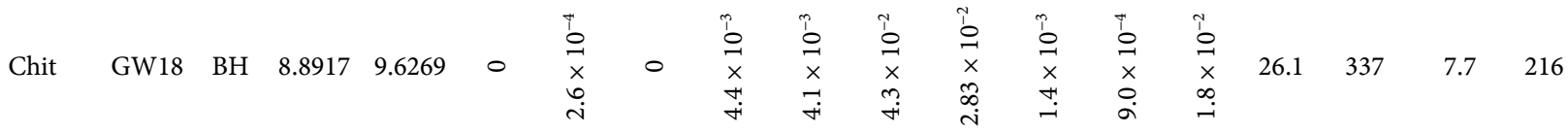

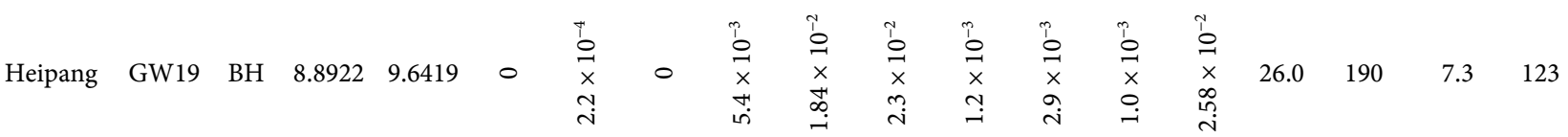

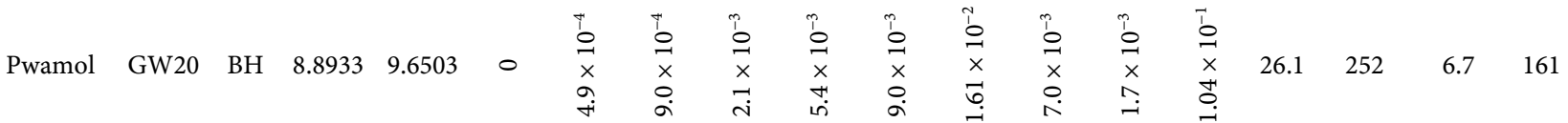

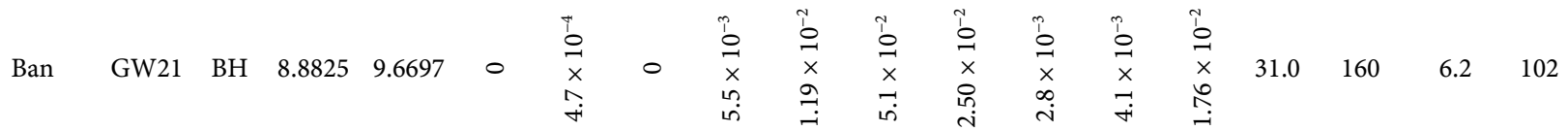

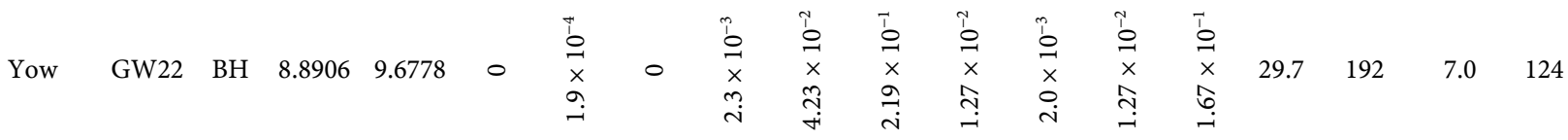

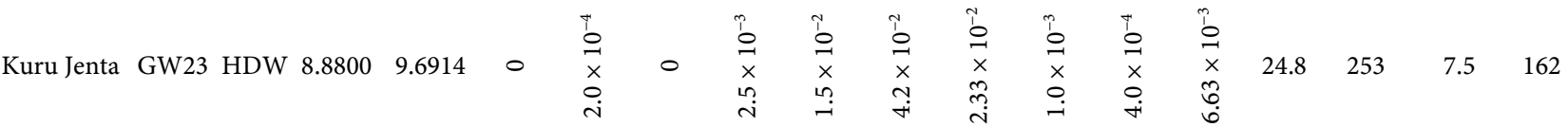




\section{Continued}

Nigerian Standard Guideline Value

Minimum

Maximum

Standard Deviation

Mean

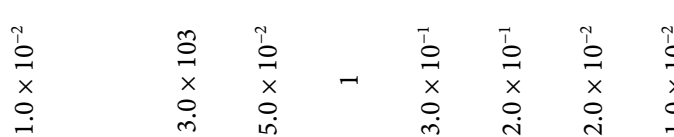

1
$\times$
0
0

$6.5-8.5 \quad 500$

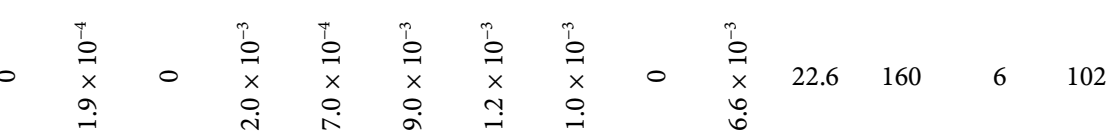

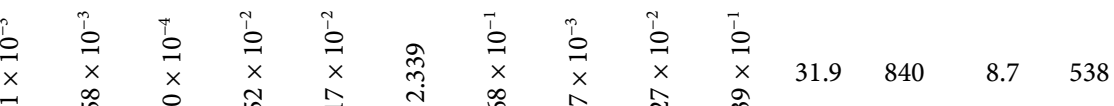

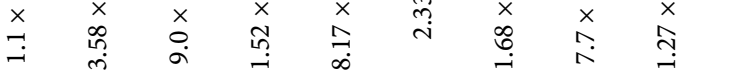

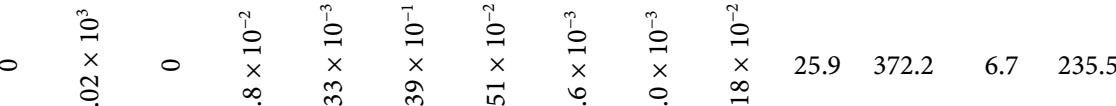$$
\text { (n) }
$$

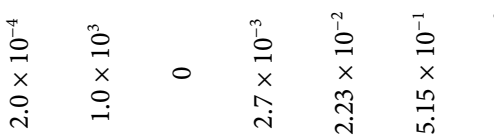

\subsection{Health Risk Assessment}

\subsubsection{Surface Water}

The hazard quotient (HQ) for adults as shown in Table 6 disclosed one (1) site within the study area that raises concern i.e. Rassal with a range of values from 0.018458 (low adverse health risk) to 1.65945 (medium non carcinogenic risk; USEPA, 1999). All the other sites sampled have HQ values between $<0.1$ to $\geq 0.1$ $<1$ implying negligible to low non carcinogenic risk (Table 3). However, considering the summation of $\mathrm{HQ}$ values for each location i.e. Hazard index (HI) for adults; Rassa1 added up to 4.98592 (high adverse health risk), Rassa2 with HI value of 1.198292 (medium adverse health risk) and Pwamol2 with HI value of 1.353486 implying medium non carcinogenic health risk. However, the HI values range from 0.095989 (negligible health risk) located at Britvic to 4.985952 (high non carcinogenic health risk; USEPA, 1999) at Rassa1 with an average HI value for adults of 0.65091 indicating low non carcinogenic health risk according to the USEPA, 1999 rating.

In addition, two (2) problematic locations (Rassaland Pwamol2) were revealed with respect to the computations for the non carcinogenic risk in children as shown in Table 7. In Rassal the range of HQ values is from 0.02568 to 2.3088 indicating negligible to medium non carcinogenic health risk (USEPA, 1999). While Pwamol2 has HQ values between 0.004187 to 1.250857 signifying negligible to medium non carcinogenic health risk on children (USEPA, 1999). In view of the additive effect of HQ's i.e. HI for children, the range is between 0.13355 to 6.936977 (low to high non carcinogenic health risk). These extreme range values are situated at Britvic and Rassal respectively as displayed in Table 7. The problematic locations include Vwei (HI value of 1.33215) implying medium adverse health risk, Bisichi1 $(\mathrm{HI}=1.3358)$ medium adverse health risk and Kuru Jenta2 
Table 6. Hazard quotient (HQ) and Hazard Index (HI) for adults in surface water.

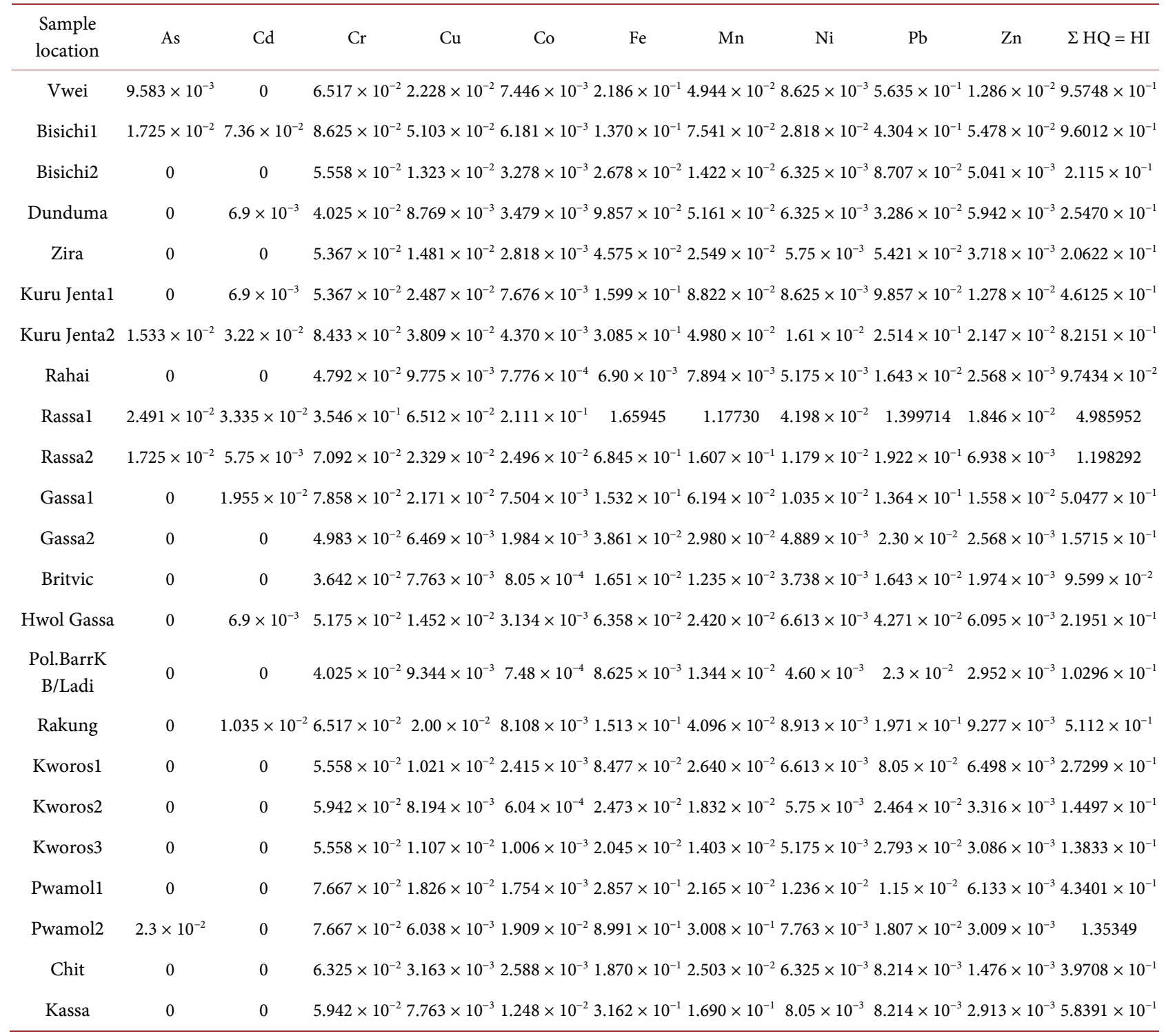

Table 7. Hazard quotient (HQ) and Hazard Index (HI) for children in surface water.

\begin{tabular}{|c|c|c|c|c|c|c|c|c|c|c|c|}
\hline $\begin{array}{c}\text { Sample } \\
\text { location }\end{array}$ & As & $\mathrm{Cd}$ & $\mathrm{Cr}$ & $\mathrm{Cu}$ & Co & $\mathrm{Fe}$ & $\mathrm{Mn}$ & $\mathrm{Ni}$ & $\mathrm{Pb}$ & $\mathrm{Zn}$ & $\Sigma \mathrm{HQ}=\mathrm{HI}$ \\
\hline Vwei & $1.333 \times 10^{-2}$ & 0 & $9.067 \times 10^{-2}$ & $3.10 \times 10^{-2}$ & $1.036 \times 10^{-2}$ & ${ }^{2} 3.041 \times 10^{-1}$ & $6.878 \times 10^{-2}$ & $1.20 \times 10^{-2}$ & $7.84 \times 10^{-1}$ & $1.789 \times 10^{-2}$ & 1.33215 \\
\hline Bisichi1 & $2.4 \times 10^{-2}$ & $1.024 \times 10^{-1}$ & ${ }^{1} 1.20 \times 10^{-1}$ & $7.10 \times 10^{-2}$ & $8.60 \times 10^{-3}$ & $1.906 \times 10^{-1}$ & $1.049 \times 10^{-1}$ & $3.92 \times 10^{-2}$ & $5.99 \times 10^{-1}$ & $7.621 \times 10^{-2}$ & 1.33582 \\
\hline Bisichi2 & 0 & 0 & $7.733 \times 10^{-2}$ & $1.84 \times 10^{-2}$ & $4.56 \times 10^{-3}$ & $3.726 \times 10^{-2}$ & $1.978 \times 10^{-2}$ & $8.80 \times 10^{-3}$ & $1.211 \times 10^{-1}$ & $7.013 \times 10^{-3}$ & $2.943 \times 10^{-1}$ \\
\hline Dunduma & 0 & $9.60 \times 10^{-3}$ & $5.60 \times 10^{-2}$ & $1.22 \times 10^{-2}$ & $4.84 \times 10^{-3}$ & $1.371 \times 10^{-1}$ & $7.181 \times 10^{-2}$ & $8.80 \times 10^{-3}$ & $4.571 \times 10^{-2}$ & $8.267 \times 10^{-3}$ & $3.544 \times 10^{-1}$ \\
\hline Zira & 0 & 0 & $7.467 \times 10^{-2}$ & $2.06 \times 10^{-2}$ & $3.92 \times 10^{-3}$ & $6.366 \times 10^{-2}$ & $3.547 \times 10^{-2}$ & $8.00 \times 10^{-3}$ & $7.542 \times 10^{-2}$ & $5.173 \times 10^{-3}$ & $2.869 \times 10^{-1}$ \\
\hline Kuru Jenta 1 & 0 & $9.60 \times 10^{-3}$ & $7.467 \times 10^{-2}$ & $3.46 \times 10^{-2}$ & $1.068 \times 10^{-2}$ & $2.225 \times 10^{-1}$ & $1.227 \times 10^{-1}$ & $1.20 \times 10^{-2}$ & $1.371 \times 10^{-1}$ & $1.779 \times 10^{-2}$ & $6.417 \times 10^{-1}$ \\
\hline Kuru Jenta2 & $2.133 \times 10^{-2}$ & ${ }^{2} 4.48 \times 10^{-2}$ & $1.173 \times 10^{-1}$ & $5.30 \times 10^{-2}$ & $6.08 \times 10^{-3}$ & $4.291 \times 10^{-1}$ & $6.929 \times 10^{-2}$ & $2.24 \times 10^{-2}$ & $3.497 \times 10^{-1}$ & $2.988 \times 10^{-2}$ & 1.14296 \\
\hline Rahai & 0 & 0 & $6.667 \times 10^{-2}$ & $1.36 \times 10^{-2}$ & $1.08 \times 10^{-3}$ & $9.6 \times 10^{-3}$ & $1.098 \times 10^{-2}$ & $7.20 \times 10^{-3}$ & $2.286 \times 10^{-2}$ & $3.573 \times 10^{-3}$ & $1.356 \times 10^{-1}$ \\
\hline Rassa1 & $3.467 \times 10^{-2}$ & $24.64 \times 10^{-2}$ & $4.933 \times 10^{-1}$ & $9.06 \times 10^{-2}$ & $2.937 \times 10^{-1}$ & 2.3088 & 1.6380 & $5.84 \times 10^{-2}$ & 1.9474 & $2.568 \times 10^{-2}$ & 6.93697 \\
\hline
\end{tabular}




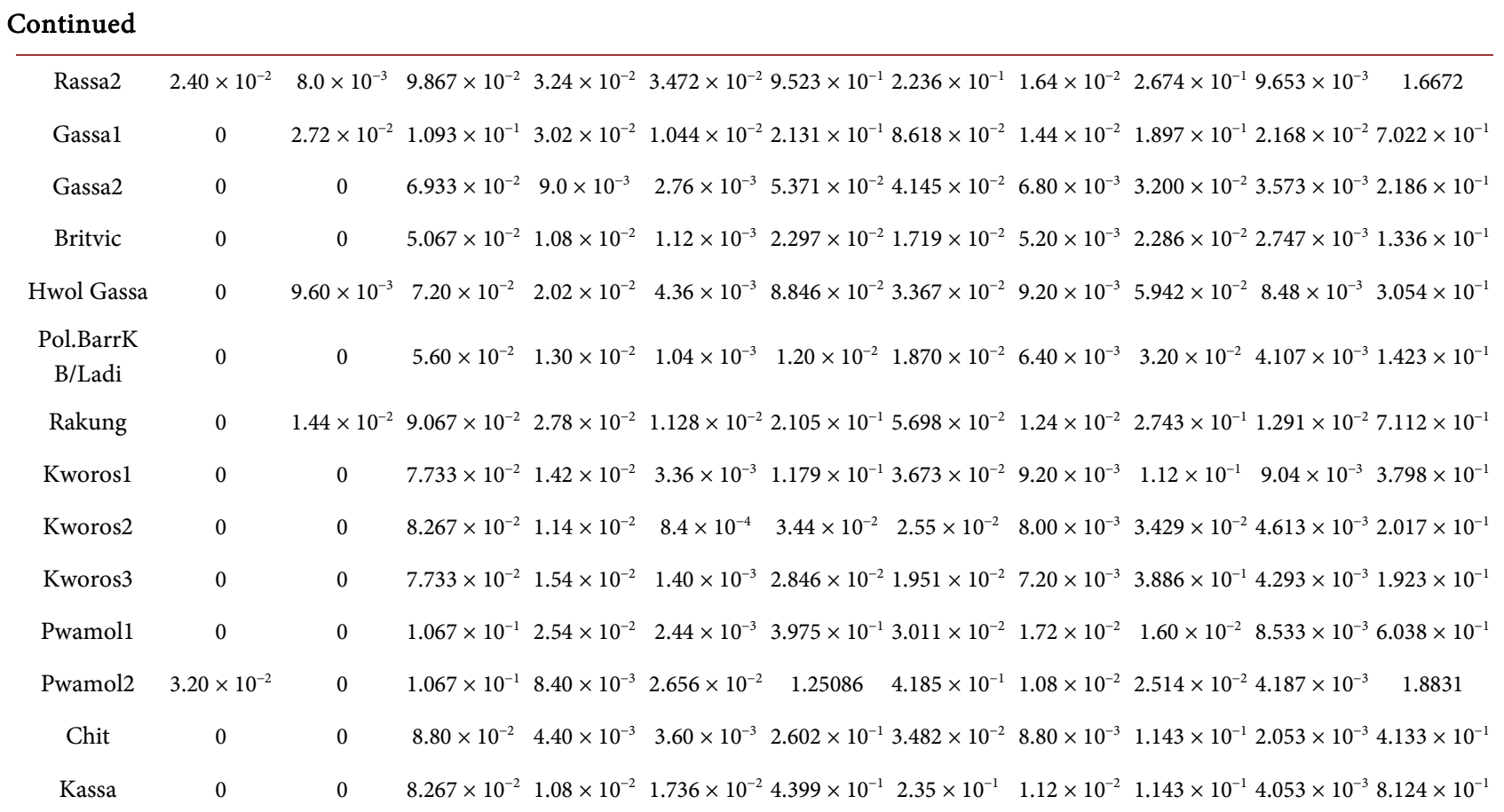

$(\mathrm{HI}=1.14296$ indicating medium adverse health risk). Others include Rassa1 where $\mathrm{HI}$ is 6.936977 implying high adverse health risk, Rassa2 $(\mathrm{HI}=1.667189)$ meaning medium adverse health risk as well as Pwamol2 with HI value of 1.88311 suggesting medium non carcinogenic health risk. However, the average HI value in this case is 0.90561 implying low non carcinogenic health risk.

The cancer risk levels of As for adults through exposure to the surface water sources within the study area range from 7.8E-05 (medium cancer risk; USEPA, 1999) to 1.0E-04 (high cancer risk) located at Rassa2 and Pwamol2 respectively. On the average, the cancer risk of As for adults is $8.1 \mathrm{E}-05$ (medium cancer risk) implying an approximate number of eight (8) people in a hundred thousand. In the same way, the cancer risk levels of As for children ranges from 6.0E-05 (medium cancer risk level; USEPA, 1999) to 1.0E-04 (high cancer risk) situated at Vwei and Kuru Jenta2 respectively with an average value of 1.1E-04 (high cancer risk) i.e. one (1) person in a total of 10,000 people. This is displayed in Table 8 below.

The carcinogenic risk of cadmium (Cd) for adults range from 6.3E-06 (low cancer risk; USEPA, 1999) to 1.4E-05 (medium cancer risk) observed in Rassa1 and Bisichil respectively. The average cancer risk level in this case is $4.1 \mathrm{E}-06$ (low cancer risk) meaning four (4) people in a million. Similarly, the cancer risk of $\mathrm{Cd}$ in children range from 8.8E-06 (low cancer risk) to 2.0E-05 (medium cancer risk; USEPA, 1999) at Rassal and Bisichi1, with an overall average of $5.8 \mathrm{E}-06$ (low cancer risk) implying an approximate figure of six (6) persons in a total of one million people.

The cancer risk of chromium $(\mathrm{Cr})$ in adults range from $9.4 \mathrm{E}-03$ to $1.0 \mathrm{E}-02$ (Table 8) considered as very high cancer risk (USEPA, 1999) with an average 
Table 8. Cancer risk for adults and children in surface water.

\begin{tabular}{|c|c|c|c|c|c|c|}
\hline \multicolumn{4}{|c|}{ CANCER (ADULT) } & \multicolumn{3}{|c|}{ CANCER (CHILDREN) } \\
\hline Sample location & As & $\mathrm{Cd}$ & $\mathrm{Cr}$ & As & $\mathrm{Cd}$ & $\mathrm{Cr}$ \\
\hline Vwei & $4.3 \mathrm{E}-05$ & 0 & $8.0 \mathrm{E}-03$ & $6.0 \mathrm{E}-05$ & 0 & $1.1 \mathrm{E}-02$ \\
\hline Bisichil & $7.8 \mathrm{E}-05$ & $1.4 \mathrm{E}-05$ & $1.2 \mathrm{E}-02$ & $1.1 \mathrm{E}-04$ & $2.0 \mathrm{E}-05$ & $1.5 \mathrm{E}-02$ \\
\hline Bisichi2 & 0 & 0 & $6.8 \mathrm{E}-03$ & 0 & 0 & $9.5 \mathrm{E}-03$ \\
\hline Dunduma & 0 & $1.3 \mathrm{E}-06$ & $5.0 \mathrm{E}-03$ & 0 & $1.8 \mathrm{E}-06$ & $6.9 \mathrm{E}-03$ \\
\hline Zira & 0 & 0 & $6.6 \mathrm{E}-03$ & 0 & 0 & $9.2 \mathrm{E}-03$ \\
\hline Kuru Jenta 1 & 0 & $1.3 \mathrm{E}-06$ & $6.6 \mathrm{E}-03$ & 0 & $1.8 \mathrm{E}-06$ & $9.2 \mathrm{E}-03$ \\
\hline Kuru Jenta2 & $6.9 \mathrm{E}-05$ & $6.1 \mathrm{E}-06$ & $1.0 \mathrm{E}-02$ & $1.0 \mathrm{E}-04$ & $8.5 \mathrm{E}-06$ & $1.4 \mathrm{E}-02$ \\
\hline Rahai & 0 & 0 & $5.9 \mathrm{E}-03$ & 0 & 0 & $8.2 \mathrm{E}-03$ \\
\hline Rassal & $1.2 \mathrm{E}-04$ & $6.3 \mathrm{E}-06$ & $4.4 \mathrm{E}-02$ & $1.6 \mathrm{E}-04$ & $8.8 \mathrm{E}-06$ & $6.1 \mathrm{E}-02$ \\
\hline Rassa2 & $7.8 \mathrm{E}-05$ & $1.1 \mathrm{E}-06$ & $8.7 \mathrm{E}-03$ & $1.1 \mathrm{E}-04$ & $1.5 \mathrm{E}-06$ & $1.2 \mathrm{E}-02$ \\
\hline Gassal & 0 & $3.7 \mathrm{E}-06$ & $1.0 \mathrm{E}-02$ & 0 & $5.2 \mathrm{E}-06$ & $1.4 \mathrm{E}-02$ \\
\hline Gassa2 & 0 & 0 & $6.1 \mathrm{E}-03$ & 0 & 0 & $8.5 \mathrm{E}-03$ \\
\hline Britvic & 0 & 0 & $4.5 \mathrm{E}-03$ & 0 & 0 & $6.2 \mathrm{E}-03$ \\
\hline Hwol Gassa & 0 & $1.3 \mathrm{E}-06$ & $6.4 \mathrm{E}-03$ & 0 & $1.8 \mathrm{E}-06$ & $8.9 \mathrm{E}-03$ \\
\hline Pol.BarrK B/Ladi & 0 & 0 & $5.0 \mathrm{E}-03$ & 0 & 0 & $6.9 \mathrm{E}-03$ \\
\hline Rakung & 0 & $2.0 \mathrm{E}-06$ & $8.0 \mathrm{E}-03$ & 0 & $2.7 \mathrm{E}-06$ & $1.1 \mathrm{E}-02$ \\
\hline Kworos1 & 0 & 0 & $6.8 \mathrm{E}-03$ & 0 & 0 & $9.5 \mathrm{E}-03$ \\
\hline Kworos2 & 0 & 0 & $7.3 \mathrm{E}-03$ & 0 & 0 & $1.0 \mathrm{E}-02$ \\
\hline Kworos3 & 0 & 0 & $6.8 \mathrm{E}-03$ & 0 & 0 & $9.5 \mathrm{E}-03$ \\
\hline Pwamol1 & 0 & 0 & $9.4 \mathrm{E}-03$ & 0 & 0 & $1.3 \mathrm{E}-02$ \\
\hline Pwamol2 & $1.0 \mathrm{E}-04$ & 0 & $9.4 \mathrm{E}-03$ & $1.4 \mathrm{E}-04$ & 0 & $1.3 \mathrm{E}-02$ \\
\hline Chit & 0 & 0 & $7.8 \mathrm{E}-03$ & 0 & 0 & $1.1 \mathrm{E}-02$ \\
\hline Kassa & 0 & 0 & $7.3 \mathrm{E}-03$ & 0 & 0 & $1.0 \mathrm{E}-02$ \\
\hline Mean value & $8.1 \mathrm{E}-05$ & $4.1 \mathrm{E}-06$ & $9.1 \mathrm{E}-03$ & $1.1 \mathrm{E}-04$ & $5.8 \mathrm{E}-06$ & $1.3 \mathrm{E}-02$ \\
\hline
\end{tabular}

value of 9.1E-03 (very high cancer risk) meaning nine (9) persons in one thousand people. A similar situation is seen in the cancer risk of $\mathrm{Cr}$ in children where the range is from $9.5 \mathrm{E}-03$ to $1.0 \mathrm{E}-02$ as shown in Table 8 , with an average value of $1.3 \mathrm{E}-02$ (very high cancer risk) i.e. one (1) person in a pool of one hundred.

\subsubsection{Groundwater}

The calculated HQ for adults in groundwater on behalf of all the metals considered range from $0-0.291333$ indicating negligible to low non carcinogenic risk. Similarly, the HI for the adults in groundwater range from 0.066046 (negligible non cancer risk) to 0.377082 (low non carcinogenic risk) as shown in Table 9 which does not pose a serious health concern. Similarly, the HQ for children in groundwater range from 0 to 0.405333 signifying negligible to low non cancer 
Table 9. Hazard Quotient (HQ), Hazard Index (HI) for adults in groundwater.

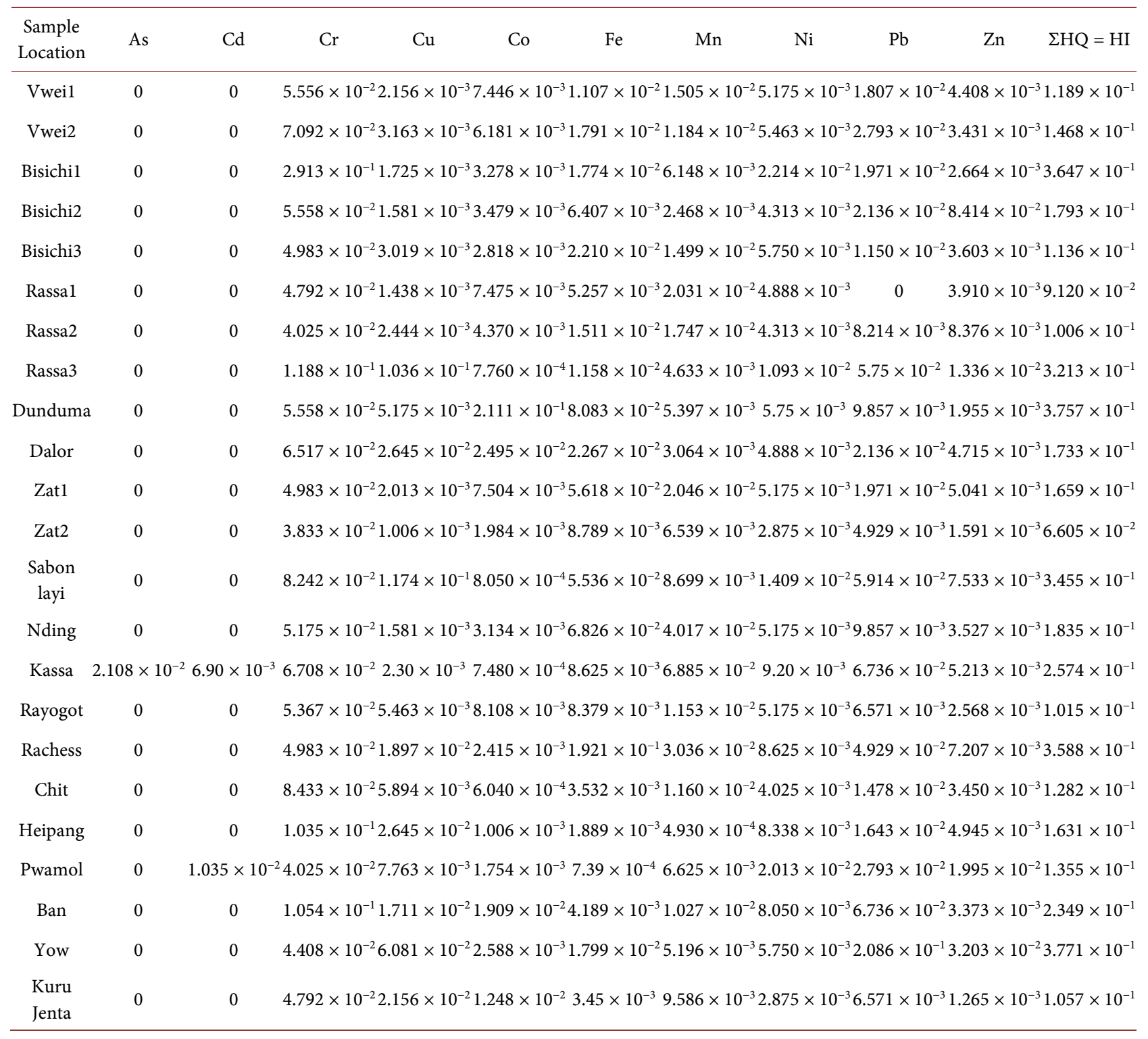

health risk. Also, the HI values range from 0.09189 to 0.524636 (Table 10) denoting negligible to low non cancer risk on children (USEPA, 1999).

From Table 11, the cancer risk of arsenic for adults through exposure to groundwater within the study area is situated in Kassa with a value of $9.5 \mathrm{E}-05$ (medium cancer risk; USEPA, 1999). Similarly, the cancer risk of arsenic in the case for children in groundwater is located in Kassa with a value of $1.3 \mathrm{E}-04$ (high cancer risk) as shown in Table 11.

The cancer risk of cadmium for adults in groundwater range from $1.31 \mathrm{E}-06$ to $1.97 \mathrm{E}-06$ (Table 11) located at Kassa and Pwamol respectively, implying low cancer risk (USEPA, 1999) for the two sites. Similarly, the cancer risk of cadmium for children in groundwater range from $1.82 \mathrm{E}-06$ to $2.74 \mathrm{E}-06$ at Kassa and Pwamol respectively, interpreted as low cancer risk (USEPA, 1999). 
Table 10. Hazard Quotient (HQ), Hazard Index (HI) for children in groundwater.

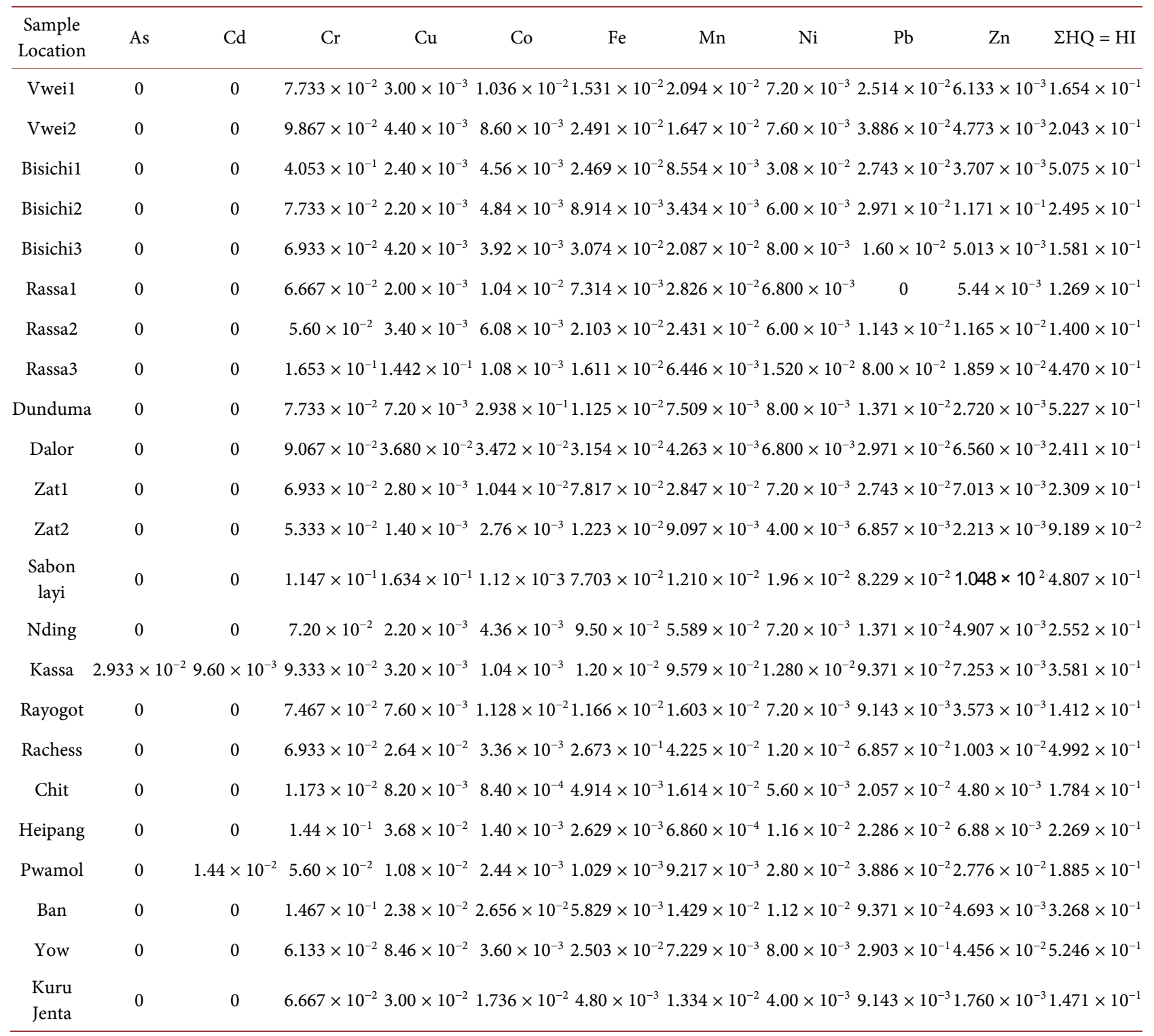

Table 11. Cancer risk for adults and children in groundwater.

\begin{tabular}{ccccccc}
\hline \multicolumn{3}{c}{ CANCER (ADULT) } & \multicolumn{3}{c}{ CANCER (CHILDREN) } \\
\hline Sample location & As & Cd & Cr & As & Cd & Cr \\
\hline Vwei1 & 0 & 0 & $6.8 \mathrm{E}-03$ & 0 & 0 & $9.5 \mathrm{E}-03$ \\
Vwei2 & 0 & 0 & $8.7 \mathrm{E}-03$ & 0 & 0 & $1.2 \mathrm{E}-02$ \\
Bisichi1 & 0 & 0 & $3.6 \mathrm{E}-02$ & 0 & 0 & $5.0 \mathrm{E}-02$ \\
Bisichi2 & 0 & 0 & $6.8 \mathrm{E}-03$ & 0 & 0 & $9.5 \mathrm{E}-03$ \\
Bisichi3 & 0 & 0 & $6.1 \mathrm{E}-03$ & 0 & 0 & $8.5 \mathrm{E}-03$ \\
Rassa1 & 0 & 0 & $5.9 \mathrm{E}-03$ & 0 & 0 & $8.2 \mathrm{E}-03$ \\
Rassa2 & 0 & 0 & $5.0 \mathrm{E}-03$ & 0 & 0 & $6.9 \mathrm{E}-03$ \\
Rassa3 & 0 & 0 & $1.5 \mathrm{E}-02$ & 0 & 0 & $2.0 \mathrm{E}-02$ \\
\hline
\end{tabular}




\begin{tabular}{ccccccc} 
Continued & \multicolumn{6}{c}{} \\
\hline Dunduma & 0 & 0 & $6.8 \mathrm{E}-03$ & 0 & 0 & $9.5 \mathrm{E}-03$ \\
Dalor & 0 & 0 & $8.0 \mathrm{E}-03$ & 0 & 0 & $1.1 \mathrm{E}-02$ \\
Zat1 & 0 & 0 & $6.1 \mathrm{E}-03$ & 0 & 0 & $8.5 \mathrm{E}-03$ \\
Zat2 & 0 & 0 & $4.7 \mathrm{E}-03$ & 0 & 0 & $6.6 \mathrm{E}-03$ \\
Sabon layi & 0 & 0 & $1.0 \mathrm{E}-02$ & 0 & 0 & $1.4 \mathrm{E}-02$ \\
Nding & 0 & 0 & $6.4 \mathrm{E}-03$ & 0 & 0 & $8.9 \mathrm{E}-03$ \\
Kassa & $9.5 \mathrm{E}-05$ & $1.31 \mathrm{E}-06$ & $8.3 \mathrm{E}-03$ & $1.3 \mathrm{E}-04$ & $1.82 \mathrm{E}-06$ & $1.2 \mathrm{E}-02$ \\
Rayogot & 0 & 0 & $6.6 \mathrm{E}-03$ & 0 & 0 & $9.2 \mathrm{E}-03$ \\
Rachess & 0 & 0 & $6.1 \mathrm{E}-03$ & 0 & 0 & $8.5 \mathrm{E}-03$ \\
Chit & 0 & 0 & $1.0 \mathrm{E}-02$ & 0 & 0 & $1.4 \mathrm{E}-02$ \\
Heipang & 0 & 0 & $1.3 \mathrm{E}-02$ & 0 & 0 & $1.8 \mathrm{E}-02$ \\
Pwamol & 0 & $1.97 \mathrm{E}-06$ & $5.0 \mathrm{E}-03$ & 0 & $2.74 \mathrm{E}-06$ & $6.9 \mathrm{E}-03$ \\
Ban & 0 & 0 & $1.3 \mathrm{E}-02$ & 0 & 0 & $1.8 \mathrm{E}-02$ \\
Yow & 0 & 0 & $5.4 \mathrm{E}-03$ & 0 & 0 & $7.5 \mathrm{E}-03$ \\
Kuru Jenta & 0 & 0 & $5.9 \mathrm{E}-03$ & 0 & 0 & $8.2 \mathrm{E}-03$ \\
Mean value & $3.17 \mathrm{E}-05$ & $1.09 \mathrm{E}-06$ & $6.7 \mathrm{E}-03$ & $4.33 \mathrm{E}-05$ & $1.52 \mathrm{E}-06$ & $9.5 \mathrm{E}-03$ \\
\hline & & & & & &
\end{tabular}

The cancer risk of chromium for adults in groundwater ranges from $8.3 \mathrm{E}-03$ to $1.0 \mathrm{E}-02$ (Table 11) deduced as very high cancer risk (USEPA, 1999). A similar situation is seen in the case of the children where the cancer risk of chromium in groundwater ranges from $9.5 \mathrm{E}-03$ to $1.1 \mathrm{E}-02$ implying very high cancer risk (USEPA, 1999).

\section{Conclusion and Recommendations}

According to the findings in this research, the human health risk levels associated with the consumption/irrigation domestic use of the surface water resources is high as compared with the groundwater sources. However, heavy metal movement through these two sources (ground and surface water) is expected over time. This would pose serious health problems (carcinogenic and/or non-carcinogenic) to the residents and especially the children who consume more water per unit of body weight than adults (ENHIS, 2007).

Consequently, it is recommended that water management plans and awareness be carried out and put in place within these communities. Also, the need for government to develop methods of restoration/reclamation of the mined out areas is suggested.

\section{Acknowledgements}

The authors wish to express their sincere gratitude to TET Fund through Plateau State Polytechnic, Barkin ladi for the financial support to carry out this research. Also, the authors are thankful to the anonymous reviewers for their constructive suggestions and scrutiny. 


\section{Conflicts of Interest}

There is no conflict of interest.

\section{References}

Adamu, C. I., Nganje, T. N., \& Edet, A. (2014). Heavy Metal Contamination and Health Risk Assessment Associated with Abandoned Barite Mines in Cross River State, Southeastern Nigeria. Journal of Environmental Nanotechnology, Monitoring \& Management, 3, 10-21.

Ajayi, P. O. (2003). Comprehensive Geography for Senior Secondary Schools. Lagos: Johnson Publishers Ltd.

Apambire, W. B., Boyle, D. R., \& Michel, F. A. (1997). Geochemistry, Genesis and Health Implications of Flouriferous Groundwaters in the Upper Regions of Ghana. Environmental Geology, 33, 13-24. https://doi.org/10.1007/s002540050221

Ayantobo, O. O., Awomeso, J. A., Oluwasanya, G. O., Bada, B. S., \& Taiwo, A. M. (2014). Non-Cancer Human Health Risk Assessment from Exposure to Heavy Metals in Surface and Groundwater in Igun-Ijesha, Southwest, Nigeria. American Journal of Environmental Sciences, 10, 301-311. https://doi.org/10.3844/ajessp.2014.301.311

Elumalai, V., Brindha, K., \& Lakshmanan, E. (2017). Human Exposure Risk Assessment Due to Heavy Metals in Groundwater by Pollution Index and Multivariate Statistical Methods. A Case Study from South Africa. Water Journal, 9, 234.

ENHIS (European Environment and Health Information System) (2007). Exposure of Children to Chemical Hazards in Food. Fact Sheet No. 4.4.CODE. RPG4_Food_EXI, World Health Organization.

Fordyce, J., Smith, B., Appleton, D., Johnson, C., Smedley, P., \& Williams, L. (1999). Environmental Geochemistry and Health-Global Perspectives. British Geological Survey.

Iloeje, N. P. (1981). A New Geography of Nigeria. Lagos: Longman Nig. Ltd.

Lim, H. S., Lee, J. S., Chon, H. T., \& Sager, H. (2008). Heavy Metal Contamination and Health Risk Assessment in the Vicinity of Abandoned Songcheon Au-Ag Mine in Korea. Journal of Geochemical Exploration, 96, 223-230.

https://doi.org/10.1016/j.gexplo.2007.04.008

MacLeod, W. N., \& Berridge, N. G. (1971): Geology of the Jos Plateau. Geological Survey of Nigeria.

Montuori, P., Lama, P., Aurino, S., Naviglio, D., \& Triassi, M. (2013). Metals Loads into the Mediterranean Sea: Estimate of Sarno River Inputs and Ecological Risk. Ecotoxicology, 22, 295-307. https://doi.org/10.1007/s10646-012-1026-9

Ramadan, J. A., \& Haruna, A. I. (2018). Assessment of Heavy Metal Contamination in Surface and Groundwater Resources Using Pollution Indices in Parts of Barkin Ladi, North Central Nigeria. IOSR Journal of Applied Geology and Geophysics, 6, 25-40.

Roychowdhury, T., Tokunaga, H., \& Ando, M. (2003). Survey of Arsenic and Heavy Metals in Food Composites and Drinking Water and Estimation of Dietary Intake by the Villagers from an Arsenic Infected Area of West Bengal, India. Science of the Total Environment, 308, 15-35. https://doi.org/10.1016/S0048-9697(02)00612-5

USEPA (US Environmental Protection Agency) (1999). A Risk Assessment Multiway Exposure Spreadsheet Calculation Tool. Washington DC: United States Environmental Protection Agency.

USEPA (US Environmental Protection Agency) (2001). Baseline Human Health Risk Assessment, Vasquez Boulevard and 1-70 Superfund Site, Denver. 
http://www.epa.gov/region8/superfund/sites/VB-170-Risk.pdf.

USEPA IRIS (US Environmental Protection Agency's Integrated Risk Information System) (2011). Environmental Protection Agency Region I. Washington DC. 20460.

Wongsasuluk, P., Chotpantarat, S., Siriwong, W., \& Robson, M. (2014). Heavy Metal Contamination and Health Risk Assessment in Drinking Water from Shallow Groundwater Wells in an Agricultural Area in Ubon Ratchathani Province, Thailand. Environmental Geochemistry and Health, 36, 169-182.

https://doi.org/10.1007/s10653-013-9537-8 